\title{
Bénard-Marangoni instability in rigid rectangular containers
}

\author{
By P. C. DAUBY AND G. LEBON \\ Université de Liège, Institut de Physique B5, Sart Tilman, B 4000 Liège 1, Belgium \\ e-mail:pc.dauby@ulg.ac.be
}

(Received 7 September 1994 and in revised form 5 July 1996)

Thermocapillary convection in three-dimensional rectangular finite containers with rigid lateral walls is studied. The upper surface of the fluid layer is assumed to be flat and non-deformable but is submitted to a temperature-dependent surface tension. The realistic 'no-slip' condition at the sidewalls makes the method of separation of variables inapplicable for the linear problem. A spectral Tau method is used to determine the critical Marangoni number and the convective pattern at the threshold as functions of the aspect ratios of the container. The influence on the critical parameters of a non-vanishing gravity and a non-zero Biot number at the upper surface is also examined. The nonlinear regime for pure Marangoni convection ( $R a=0$ ) and for $P r=10^{4}, B i=0$ is studied by reducing the dynamics of the system to the dynamics of the most unstable modes of convection. Owing to the presence of rigid walls, it is shown that the convective pattern above the threshold may be quite different from that predicted by the linear approach. The theoretical predictions of the present study are in very good agreement with the experiments of Koschmieder \& Prahl (1990) and agree also with most of Dijkstra's $(1995 a, b)$ numerical results. Important differences with the analysis of Rosenblat, Homsy \& Davis (1982b) on slippery walls containers are emphasized.

\section{Introduction}

When a fluid layer is heated from below, it is well known that convection sets in after a critical temperature difference is reached between the bottom and the top of the layer. In a one-component fluid, the appearance of motion results either from gravity effects (Rayleigh-Bénard problem) or from thermocapillary phenomena (Marangoni convection). Since the celebrated papers by Bénard (1900), Rayleigh (1916), Pearson (1958) and Nield (1964), the problem of thermogravitational and thermocapillary instabilities has been of growing interest (see, for instance, Koschmieder 1993 or Platten \& Legros 1984). In most works the fluid layer is, however, assumed to be of infinite horizontal extent. This hypothesis allows the separation of variables and the linear stability problem reduces to an eigenvalue problem for a system of ordinary differential equations in the vertical coordinate. This problem has been studied thoroughly for several different boundary conditions for both the velocity and temperature at the top and the bottom of the fluid layer. However, since experiments can only take place in finite containers, the comparison between theoretical and experimental results is not always an easy task as sidewalls play an important role in small boxes.

The study of the effects of lateral walls in boxes with large aspect ratios is based on 
the 'envelope formalism' (see for instance Manneville 1990) and will not be considered here. We focus instead on instabilities in boxes with small aspect ratios. In this case, the spatial horizontal pattern of the convective cells is no longer degenerate as in infinite layers but is determined by the lateral confinement of the sidewalls.

A linear study of pure gravity-driven instability in rectangular containers with rigid horizontal and lateral walls was presented by Davis (1967). He predicted the appearance at threshold of 'finite rolls (cells with two non-zero velocity components dependent on all three spatial variables) with axes parallel to the shorter side'. These 'finite rolls' have been criticized by Davies-Jones (1970) and by Luijkx \& Platten (1981) who considered more realistic three-dimensional solutions.

The problem of thermocapillary convection in finite boxes was first considered by Rosenblat, Davis \& Homsy (1982a) and Rosenblat, Homsy \& Davis (1982b). These authors presented a linear and nonlinear study of thermoconvection due to temperature induced surface tension variations in circular and rectangular containers. However, their work is based on the 'slippery' lateral walls assumption. This boundary condition for the velocity is not realistic but enables the linear problem to be solved by using the method of separation of variables, at least for adiabatically insulated sidewalls. The nonlinear approach proposed by these authors concerns only roll-patterns but an extension was proposed by Dauby et al. (1993) who examined the possibility of the occurrence of hexagonal convective cells.

More recently Winters \& Plesser (1988), van de Vooren \& Dijkstra (1989) and Dijkstra (1992) have examined pure Marangoni convection with a 'no-slip condition' at the lateral boundaries. The velocity actually vanishes on the lateral walls and the method of separation of variables does not work. Their approaches are based on finiteelement or finite-volume methods but are restricted to two-dimensional containers.

Since we submitted the first version of this work, Dijkstra has also considered Marangoni convection in three-dimensional rigid square boxes and he has obtained interesting results by using powerful numerical methods (Dijkstra 1995a-c). The first paper $(1995 a)$ is devoted to a linear study of stability. The second is a study of nonlinear convection in square containers with aspect ratios smaller than 6 . In the third work, larger-aspect-ratio containers are investigated and hexagonal convection is shown to be possible in sufficiently large vessels. The results of Dijkstra will be compared with ours and used as validation of our method.

In the present work, we study the problem of thermocapillary convection in the general case of three-dimensional rectangular containers with realistic rigid (no-slip) lateral walls (see also Dauby \& Lebon 1994; Dauby, Lebon \& Colinet 1996). It is assumed that the upper surface of the layer is flat and non-deformable and that the lateral walls are adiabatically insulated. We consider both linear and nonlinear analyses. The dependence of the linear results on non-zero Rayleigh and Biot numbers is also examined. In the nonlinear study, the Rayleigh and Biot numbers are fixed to 0 and the Prandtl number is equal to $10^{4}$, which is a good approximation for the silicone oils used in many experiments.

The structure of the paper is as follows. In the next section, the basic equations are given. The linear analysis of the problem is developed in $\S 3$ while the nonlinear approach is treated in $\S 4$. Conclusions are drawn in the last section. Some technical points are developed in Appendices A and B. 


\section{Basic equations}

The system consists of a thin viscous fluid layer filling a rectangular container. The thickness of the layer is equal to $d$ and the length and width of the container are $a_{1} d$ and $a_{2} d$, respectively ( $a_{1}$ and $a_{2}$ are the aspect ratios). The surface tension at the upper free surface is assumed to be temperature dependent. The fluid is heated from below. It is well known that motion sets in after the vertical temperature gradient has reached a critical value.

In the reference state, the fluid is at rest and heat propagates by conduction only: the corresponding velocity and temperature fields are given by $v_{r}=0, T_{r}=T_{B}-(\Delta T / d) z$; $v=(u, v, w)$ is the velocity vector and $T$ the temperature field; subscript $r$ refers to the reference state, $T_{B}$ is the temperature of the fluid at the bottom of the box and $\Delta T$ is the temperature drop between the bottom and the top of the layer. The $z$-axis is vertical and oriented from the bottom to the top of the box.

The velocity perturbations $v=(u, v, w)$ and the temperature perturbation $\theta$ with respect to the conductive solution are governed by the Boussinesq nonlinear equations:

$$
\begin{gathered}
\nabla \cdot \boldsymbol{v}=0, \\
\operatorname{Pr}^{-1} \frac{\mathrm{d} v}{\mathrm{~d} t}=-\nabla p+R a \theta \boldsymbol{e}_{z}+\nabla^{2} \boldsymbol{v}, \\
\frac{\mathrm{d} \theta}{\mathrm{d} t}=w+\nabla^{2} \theta .
\end{gathered}
$$

Equations (2.1) (2.3) are written in dimensionless form with space, time and temperature scaled by $d, d^{2} / \kappa$ and $\Delta T$, respectively; $p$ is the dimensionless pressure and $\boldsymbol{e}_{z}$ the unit vector along the $z$-axis. The material time derivative is denoted by $\mathrm{d} / \mathrm{d} t=\partial / \partial t+v \cdot \nabla$. The Rayleigh number $R a$ is defined by

$$
R a=\frac{\alpha g \Delta T d^{3}}{\kappa \nu},
$$

where $\alpha$ is the coefficient of volumetric expansion and $g$ the acceleration due to gravity; $\nu$ is the kinematic viscosity of the fluid and $\kappa$ its heat diffusivity. The Prandtl number $\operatorname{Pr}$ is given by

$$
\operatorname{Pr}=\frac{\nu}{\kappa}
$$

The boundary conditions are the following.

The bottom of the box is rigid and perfectly heat conducting so that

$$
v=\theta=0 \quad \text { at } \quad z=0 .
$$

The upper surface of the fluid is assumed free, plane and non-deformable. The surface tension $\xi$ is supposed to be a linear function of the temperature:

$$
\xi(T)=\xi\left(T_{0}\right)-\gamma\left(T-T_{0}\right)
$$

$T_{0}$ is a reference temperature, say the temperature of the ambient surroundings and $\gamma=-\partial \xi / \partial T$ is a constant which is positive for most fluids.

At the top of the layer, heat is transferred from the liquid to the ambient gas according to Newton's cooling law

$$
q=h\left(T_{s}-T_{e x t}\right)
$$


where $q$ is the normal component of the heat flux vector at the surface of the fluid, at temperature $T_{S} ; T_{e x t}$ is the constant temperature of the external medium and $h$ the heat transfer coefficient assumed to be constant. The mathematical expressions for the boundary conditions at the upper surface are (Pearson 1958; Nield 1964; Rosenblat et al. $1982 a, b)$

$$
\begin{gathered}
w=0 \quad \text { at } z=1 \\
\frac{\partial \theta}{\partial z}+B i \theta=0 \quad \text { at } \quad z=1 \\
\frac{\partial}{\partial z}\left(\begin{array}{l}
u \\
v
\end{array}\right)+M a \nabla_{h} \theta=0 \quad \text { at } \quad z=1,
\end{gathered}
$$

where $\nabla_{h}=(\partial / \partial x, \partial / \partial y)$ is the horizontal gradient and $M a$ the Marangoni number defined by

$$
M a=\frac{\gamma \Delta T d}{\rho \nu \kappa} .
$$

The symbol $B i=h d / \lambda$ stands for the non-dimensional heat transfer coefficient (the so-called Biot number), with $\lambda$ the heat conductivity of the fluid. In (2.12), $\rho$ is the mass density.

The sidewalls are adiabatically insulated and rigid. The corresponding boundary conditions for velocity and temperature are

$$
\begin{aligned}
& u=v=w=\frac{\partial \theta}{\partial x}=0 \quad \text { at } \quad x=0, a_{1}, \\
& u=v=w=\frac{\partial \theta}{\partial y}=0 \quad \text { at } \quad y=0, a_{2} .
\end{aligned}
$$

\section{Linear stability problem}

The linear stability problem consists in determining the critical value of the Marangoni or Rayleigh number above which convection sets in. This is achieved by first linearizing equations (2.1)-(2.3). The boundary conditions (2.6), (2.9)-(2.11), (2.13) and (2.14), which are linear, keep the same form. Then, an exponential time dependence of the form $\exp (\sigma t)$ for all the variables is introduced in the equations, which results in an eigenvalue problem for the Marangoni number, the Rayleigh number or the growth rate $\sigma$ :

$$
\begin{gathered}
\boldsymbol{\nabla} \cdot \boldsymbol{v}=0 \\
\nabla^{2} \boldsymbol{v}-\boldsymbol{\nabla} p+\operatorname{Ra} \theta \boldsymbol{e}_{z}=\operatorname{Pr}^{-1} \sigma v \\
\nabla^{2} \theta+w=\sigma \theta
\end{gathered}
$$

Since we are mainly interested in surface-tension driven instability, we will consider in this section that the Rayleigh number is fixed and we will determine the critical Marangoni number characterizing marginal stability. Moreover, the principle of exchange of stability is assumed to hold. It is supposed that the growth rate of the most dangerous mode passes from real negative to real positive values as the control parameter is increased above its critical value. This assumption has been shown to be valid for pure buoyancy instability (Pellew \& Southwell 1940) owing to the selfadjointness of the relevant equations. Exchange of stability was also proved numerically 
to hold in the case of pure thermocapillary (Vidal \& Acrivos 1966) and coupled buoyancy and thermocapillary (Takashima 1970) instabilities in infinite boxes. For finite containers, no demonstration has been proposed up to now. Nevertheless, Rosenblat et al. $(1982 a, b)$ have taken this principle for granted in the case of slippery lateral boundaries. Moreover, numerical works by Dijkstra $(1992,1995 b)$ show that no Hopf bifurcations occur at threshold in rigid containers. So the assumption of exchange of stability is made here and both the real and imaginary parts of the perturbations growth rate are assumed to vanish at the threshold.

In their papers, Rosenblat et al. use the method of separation of variables to study the linear Marangoni instability in a container with slippery walls. As shown by Pellew \& Southwell (1940), this method cannot be applied when the sidewalls are rigid and a numerical resolution of the eigenvalue problem must be considered.

\subsection{The numerical method}

The numerical method to be used here is the so-called spectral Tau method (Canuto et al. 1988), which is a refinement of the well-known Galerkin method (Finlayson 1972). Following this approach, the unknown fields are expanded in series of trial functions which form a complete set and satisfy some of the boundary conditions, the so-called 'essential' boundary conditions. Truncated series are introduced in the field equations as well as in the 'natural' boundary conditions which are not a priori satisfied by the trial functions. Then these equations and natural boundary conditions are projected on the same trial functions, i.e. the equations and natural boundary conditions are multiplied by the trial functions before being integrated over the fluid volume. The purpose of this procedure is to replace the set of differential equations by a set of algebraic equations.

In the present problem, the unknowns $v(u, v, w)$ and $\theta$ are written in the form

$$
\left(\begin{array}{l}
v \\
\theta
\end{array}\right)=\sum_{i=1}^{N_{x}} \sum_{j=1}^{N_{y}} \sum_{k=1}^{N_{z}} A_{i j k}\left(\begin{array}{c}
\boldsymbol{v}_{i j k}^{X} \\
0
\end{array}\right)+\sum_{i=1}^{N_{x}} \sum_{j=1}^{N_{y}} \sum_{k=1}^{N_{z}} B_{i j k}\left(\begin{array}{c}
\boldsymbol{v}_{i j k}^{Y} \\
0
\end{array}\right)+\sum_{i=1}^{N_{x}} \sum_{j=1}^{N_{y}} \sum_{k=1}^{N_{z}} C_{i j k}\left(\begin{array}{c}
0 \\
\theta_{i j k}
\end{array}\right),
$$

where $N_{x}, N_{y}$ and $N_{z}$ are integers; $v_{i j k}^{X}=\left(u_{i j k}^{X}, 0, w_{i j k}^{X}\right), \boldsymbol{v}_{i j k}^{Y}=\left(0, v_{i j k}^{Y}, w_{i j k}^{Y}\right)$ and $\theta_{i j k}$ are trial functions which are specified in Appendix $\mathrm{A} ; A_{i j k}, B_{i j k}$ and $C_{i j k}$ are unknown constants. The total number of trial functions or degrees of freedom is thus given by $3 \times N_{x} \times N_{y} \times N_{z}$.

The unknown pressure $p$ is not given an explicit decomposition because the pressure gradient disappears in the final equations, thanks to integration by parts and boundary conditions.

The expressions $v_{i j k}^{X}=\left(u_{i j k}^{X}, 0, w_{i j k}^{X}\right)$ and $v_{i j k}^{Y}=\left(0, v_{i j k}^{Y}, w_{i j k}^{Y}\right)$ correspond to the 'finite rolls' solutions of Davis (1967), i.e. to modes of convection for which one of the two horizontal components of the velocity vanishes but whose non-zero components are actually functions of the three spatial coordinates. The upper indices $X$ and $Y$ define the ' $x$-rolls' and ' $y$-rolls' of Davis which are parallel to the $y$ - and $x$-axes, respectively. Let us mention that these finite rolls have been criticized by different authors (DaviesJones 1970; Luijkx \& Platten 1981; Platten \& Legros 1984) who showed that these modes of convection can never be exact solutions of the problem. In the present work, we do not consider separately $x$-rolls and $y$-rolls, as done by Davis, but we decompose the solution into a sum of such rolls.

The precise form of the trial functions and some further details on how to obtain the algebraic eigenvalue problem are given in Appendix A. Note only that, owing to the symmetry between the 'length' and the 'width' of the box, the solutions of the eigenvalue problem may be separated into four classes which are characterized by the 
parity of the unknown fields with respect to the coordinates $x$ and $y$. The four classes will be denoted $E E, E O, O E$ and $O O$. The first and second letter of each of these symbols respectively refers to the parity of the $x$ - and $y$-dependences of the temperature field. Note also that the normalization condition that has been used in the determination of the algebraic eigenvectors is written as

$$
\sum_{i}\left|\theta_{i}\right|=1,
$$

where $\theta_{i}$ represents the values of the temperature field at the nine points chosen at middepth of the container and with $x$-coordinates equal to $0,-\frac{1}{2} a_{1},-a_{1}$ and $y$-coordinates equal to $0,-\frac{1}{2} a_{2},-a_{2}$. This normalization condition is of importance for the nonlinear analysis developed in $\S 4$.

\subsection{Results}

Before considering three-dimensional containers, we have checked our method by comparing our two-dimensional results with previous approaches based on other techniques.

Table 1 gives a comparison with the works of Winters \& Plesser (1988), van de Vooren \& Dijkstra (1989) and Dijkstra (1992). The convergence of our algorithm is also examined. The agreement between the present work and the previous ones is excellent as soon as $N_{x} \times N_{z}$ is equal to $5 \times 5$. The convergence is also very satisfactory since the difference between the results with $N_{x} \times N_{z}=8 \times 8$ and those with $N_{x} \times N_{z}=5 \times 5$ is always smaller than $0.10 \%$. The fast convergence with a rather small number of trial functions is one of the principal improvements of the present work for two-dimensional containers with respect to the linear approaches given by Winters \& Plesser (1988), van de Vooren \& Dijkstra (1989) and Dijkstra (1992). Clearly, the decomposition in terms of Chebyshev polynomials is responsible for this fast convergence.

The results corresponding to the more realistic problem of three-dimensional rectangular containers are now analysed. As a preliminary, let us discuss the problem of the convergence of our method. In table 2 are found the numerical results corresponding to different values of the Rayleigh and Biot numbers and different numbers of trial functions. Two couples of aspect ratios are considered, namely $\left(a_{1}, a_{2}\right)=(4,2)$ and $\left(a_{1}, a_{2}\right)=(6,5)$. It is observed that the critical parameters evaluated with $N_{x} \times N_{y} \times N_{z}=6 \times 6 \times 6$ vary by less than $0.035 \%$ with respect to the values corresponding to $N_{x} \times N_{y} \times N_{z}=5 \times 5 \times 5$. Therefore, the next calculations are performed with $N_{x} \times N_{y} \times N_{z}=5 \times 5 \times 5$.

Table 3 presents the values of the critical Marangoni number for some aspect ratios and for zero Rayleigh and Biot numbers. Figure 1 is a three-dimensional picture of $M a_{c}$ as a function of the aspect ratios $a_{1}$ and $a_{2}(R a=B i=0)$. The calculations have been performed for aspect ratios varying by discrete steps of 0.2 . The critical Marangoni number is seen to be a globally decreasing function of the lateral dimensions of the containers. When both $a_{1}$ and $a_{2}$ become greater and greater, $M a_{c}$ tends to 79.607, which is the value found for an infinite box. However, if only one of the two aspect ratios becomes infinite, the other one being fixed, then the critical Marangoni number tends to a value which is greater than that for infinite boxes. A similar behaviour was found by Davis (1967) for pure gravity driven convection. It is also interesting to note that the decrease of $M a_{c}$ consists of portions of concave surfaces. The intersections of these portions describe, in fact, the appearance or disappearance of convective cells, or, more precisely, these intersections correspond to the transitions from one class of parity for the eigenmode $(E E, E O, O E, O O)$ to another. 


$\begin{array}{lrcccccccl}a_{1} & B i & \text { W-P } & \text { vdV-D } & \text { D } & \text { D-L } & \text { D - L } & \text { D }-\mathbf{L} & & \\ 1 & 1 & 212.27 & - & 212.82 & 210.99 & 211.97 & 212.19 & 0.46 & 0.10 \\ 2 & 1 & 145.76 & - & 146.41 & 145.65 & 145.74 & 145.75 & 0.067 & 0.0069 \\ 3 & 1 & 127.00 & - & 127.54 & 126.80 & 126.95 & 126.98 & 0.12 & 0.024 \\ 4 & 20 & - & 786.67 & 792.54 & 787.62 & 788.80 & 788.84 & 0.15 & 0.0050\end{array}$

TABLE 1. Critical Marangoni number for different aspect ratios $a_{1}$ and Biot numbers $B i ; R a=0$. The results of Winters \& Plesser (1988) (W-P), van de Vooren \& Dijkstra (1989) (vdV-D) and Dijkstra (1992) (D) are reported in columns 3-5. The following three columns (D-L) present our results with the different values of $N_{x} \times N_{z}$ indicated in the first cell. The last two columns give the differences in per cent between columns $5 \times 5$ and $4 \times 4$ and columns $8 \times 8$ and $5 \times 5$ respectively. Note that the definition of the Marangoni number in van de Vooren \& Dijkstra (1989) and Dijkstra (1992) is different from ours but the values given here have been adapted to our definition.

$\begin{array}{lrrcccl}\left(a_{1}, a_{2}\right) & R a & B i & 4 \times 4 \times 4 & 5 \times 5 \times 5 & 6 \times 6 \times 6 & \Delta_{6-5} \% \\ (4,2) & 0 & 0 & 97.171 & 97.219 & 97.228 & 0.0093 \\ (4,2) & 300 & 0 & 63.766 & 63.865 & 63.871 & 0.0094 \\ (4,2) & 0 & 10 & 479.91 & 481.16 & 481.33 & 0.035 \\ (6,5) & 0 & 0 & 83.430 & 83.475 & 83.477 & 0.0024\end{array}$

TABLE 2. Convergence of the critical Marangoni number for different values of the Rayleigh and Biot numbers. The numbers $N_{x}, N_{y}, N_{z}$ of trial functions are given in the first cell of each column. The last column gives the differences in per cent between columns $6 \times 6 \times 6$ and $5 \times 5 \times 5$.

$\begin{array}{ccccccc}a_{1} \backslash a_{2} & 2 & 3 & 4 & 5 & 6 & 7 \\ 2 & 111.48 & 101.18 & 97.219 & 95.484 & 94.622 & 94.144 \\ 3 & 101.18 & 91.080 & 88.770 & 88.034 & 88.293 & 87.092 \\ 4 & 97.219 & 88.770 & 85.771 & 84.551 & 84.462 & 83.453 \\ 5 & 95.484 & 88.034 & 84.551 & 83.772 & 83.475 & 82.484 \\ 6 & 94.622 & 88.293 & 84.462 & 83.475 & 83.178 & 82.184 \\ 7 & 94.144 & 87.092 & 83.453 & 82.484 & 82.184 & 81.976\end{array}$

TABLE 3. Values of the critical Marangoni number for different aspect ratios and for $R a=B i=0$ $\left(N_{x} \times N_{y} \times N_{z}=5 \times 5 \times 5\right)$.

The study of the number and the form of the convection cells is performed by using figure 2. This picture is a map of the modes of convection at threshold: to each pair of aspect ratios $\left(a_{1}, a_{2}\right)$ is associated a small square whose relative darkness is determined by the class $O O, O E, E O$ or $E E$ to which the critical solution pertains. Clearly, the instability is degenerate for boxes whose aspect ratios are located on the intersection of zones of different darkness since, in this case, several distinct convective modes become simultaneously unstable. The linear results for square containers are presented in figure 3 . The different portions of the decreasing $M a_{c}$ function are clearly exhibited as well as the mode switching. Note that not only the smallest eigenvalue $M a_{c}$ has been plotted, but also some other curves corresponding to the successive eigenvalues.

A precise interpretation of the map of convective patterns at threshold (figure 2) is not completely straightforward and requires the explicit representation of the convective cells for at least some values of the aspect ratios. As an example, let us 


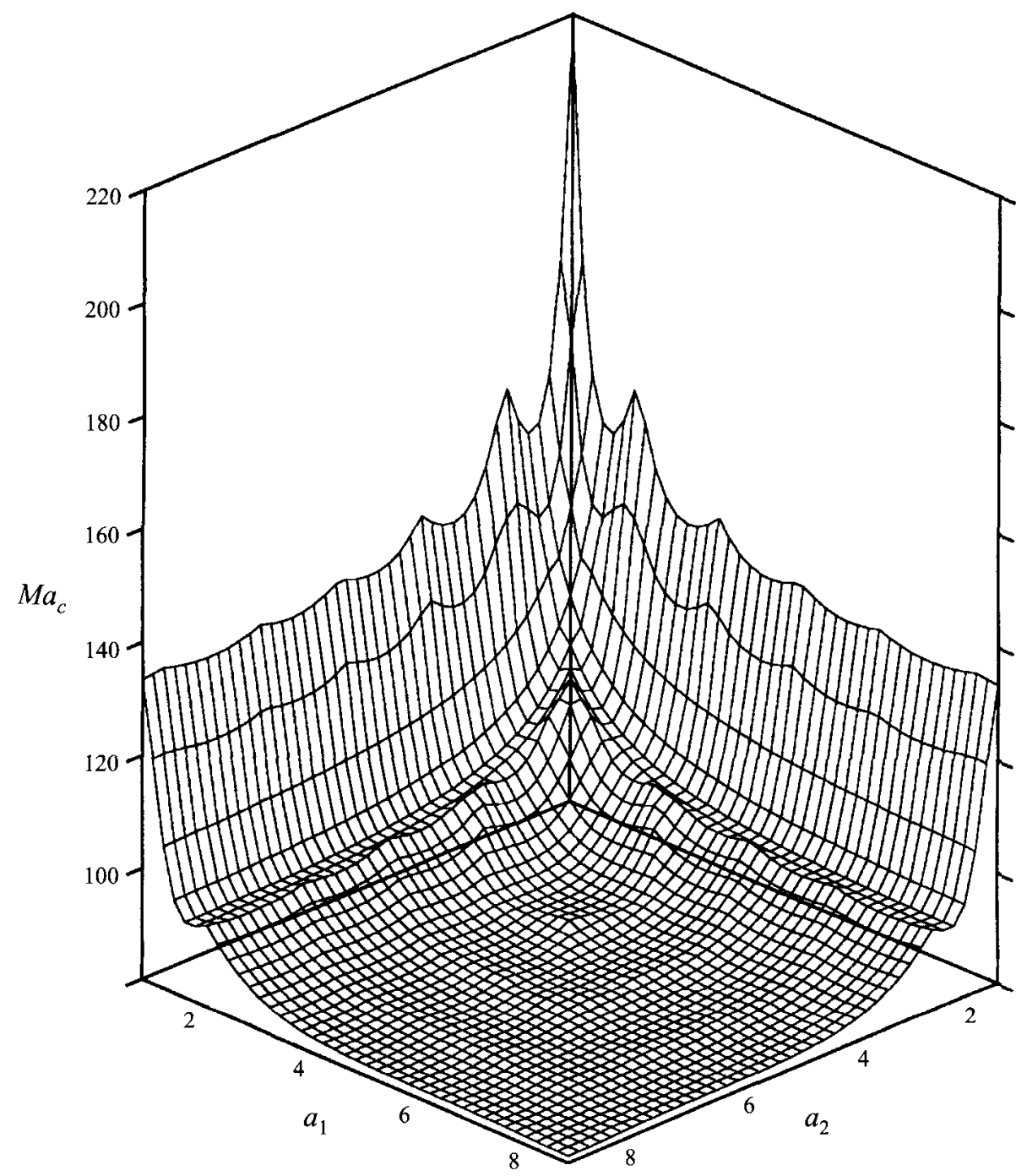

FIGURE 1. Critical Marangoni number as a function of the aspect ratios for $R a=B i=0$.

consider a container with $\left(a_{1}, a_{2}\right)=(6.6,5.2)$ in the case of zero Rayleigh and Biot numbers. For this container, the solution belongs to the $E E$ class as shown in figure 2. Various features of the flow in this box at threshold are found in figure 4. We show the horizontal velocity vector and the temperature field at the top surface and also the vertical velocity $w$ at mid-depth of the layer. We then show the velocity components $u$ and $w$ in the vertical plane $y=3.9$ as well as the velocity component $v$ in this plane. It is clearly seen that convection sets in in the form of four rolls with axes parallel to the shorter sides of the container.

To analyse figure 2, note first that this picture is 'symmetric' with respect to the line $a_{1}=a_{2}$ and only the region with $a_{1} \leqslant a_{2}$ will be studied in detail. Furthermore, figure 2 is seen to be made up of different strips with distinct colours. We will now see that each strip corresponds to a flow pattern taking the form of convective rolls which are usually parallel to the shorter sides of the box. For square boxes, rolls parallel to both sides may possibly combine to produce a more symmetric structure. 


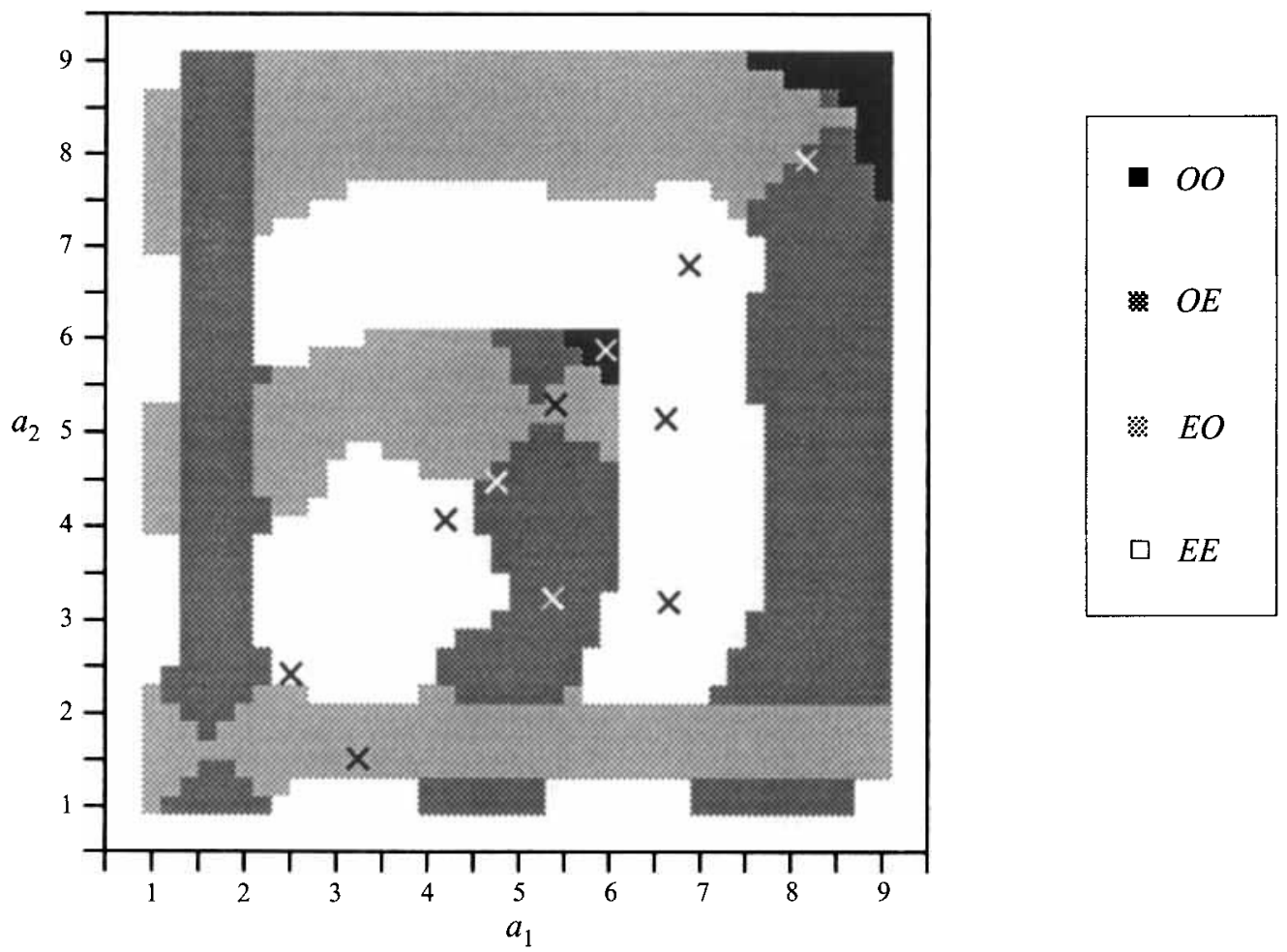

FIGURE 2. Map of the convective modes at threshold for $R a=B i=0$. Crosses indicate boxes which are considered in detail in the nonlinear analysis $(\$ 4.4)$.

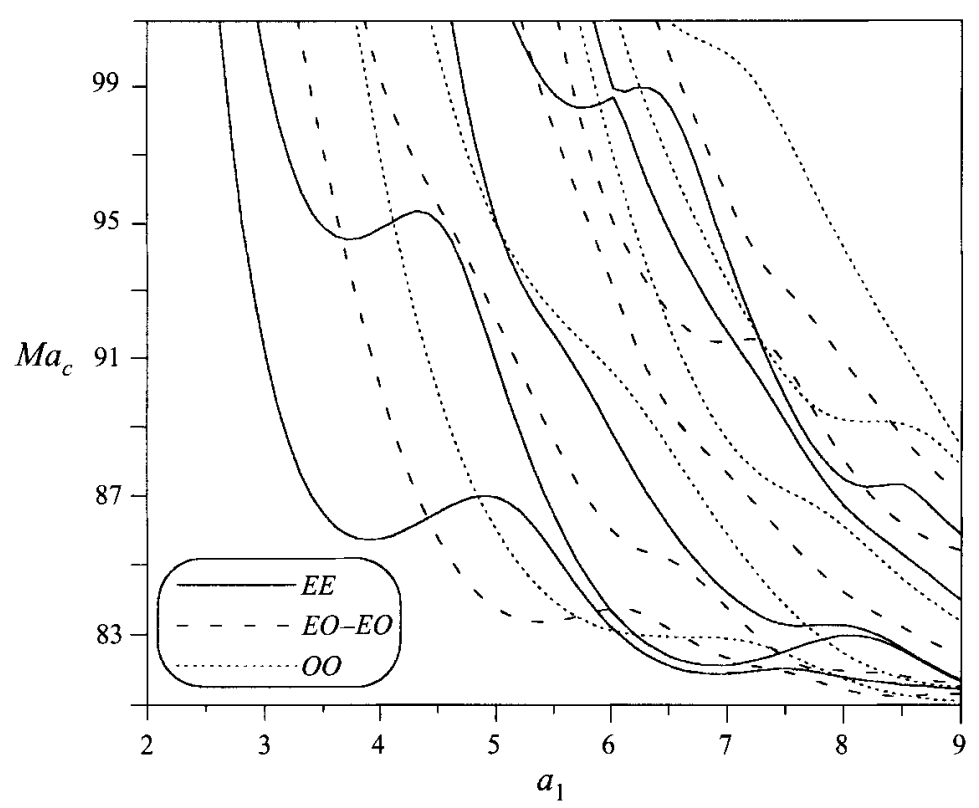

FIGURE 3. Critical Marangoni number as a function of the aspect ratio for square boxes $(R a=B i=0)$. The curves corresponding to several successive eigenvalues are also plotted and the parity of the eigenmodes is indicated. 

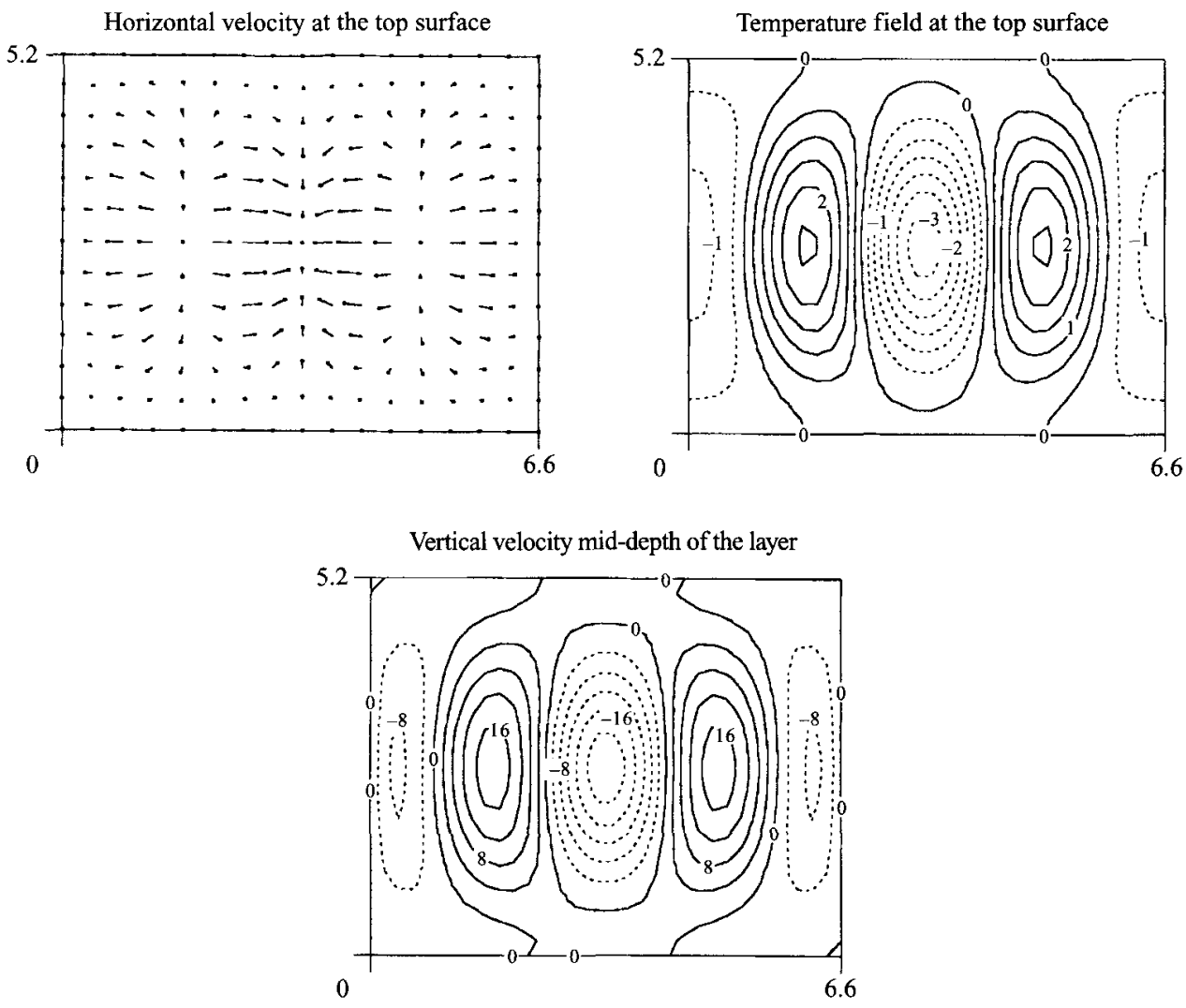

Velocity components $u$ and $w$ in the plane $y=3.9$
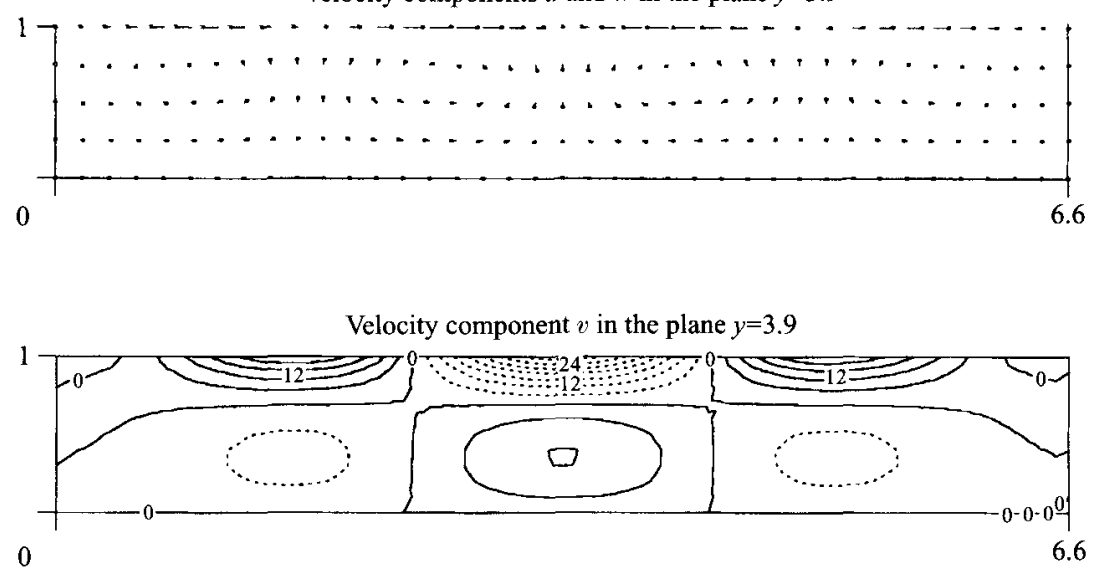

Figure 4. Representations of the flow at threshold in a box of aspect ratios $\left(a_{1}, a_{2}\right)=(6.6,5.2)$. The Rayleigh and Biot numbers are zero.

First, we consider boxes for which the aspect ratios are 'quite' different; the case of square or quasi-square containers will be studied later on. From figure 4 and from representations of the flow for other boxes with aspect ratios in the same $E E$ zone, it may be inferred that the white strip in the neighbourhood of $a_{1}=6.2$ in figure 2 corresponds to a convective structure in the form of four rolls parallel to the shorter sides of the container. Similarly, the dark grey strip centred on $a_{1}=4.8$ and the white 
(a)

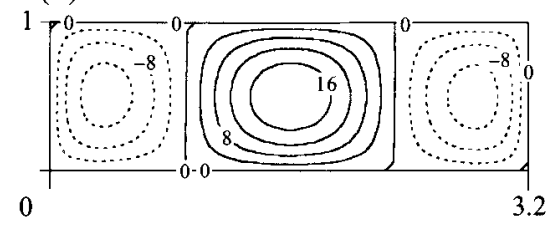

(b)

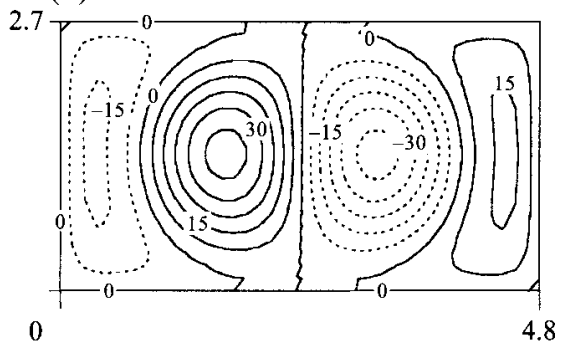

(c)

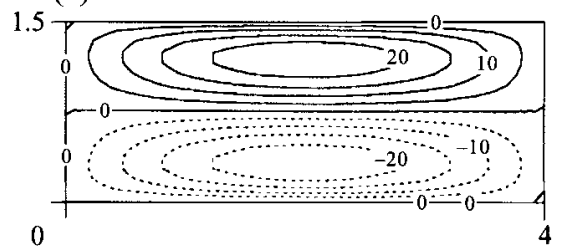

$(d)$

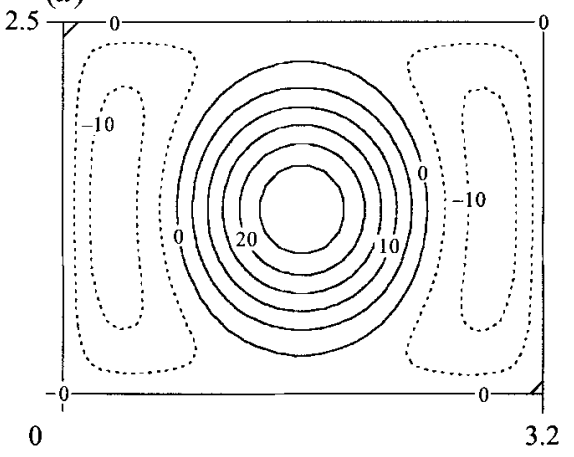

(e)

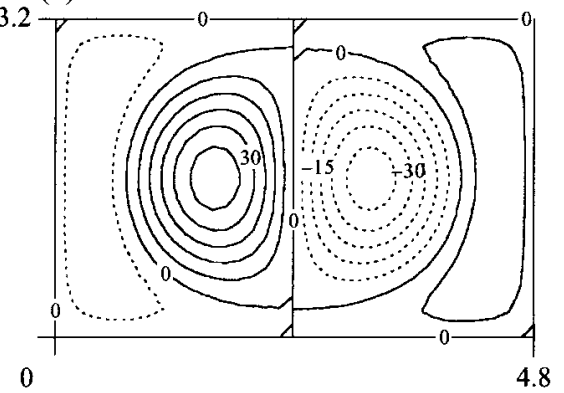

FIGURE 5. Representation of the flow at threshold for several rectangular containers $(R a=B i=0)$. The iso-values of the vertical velocity $w$ at mid-depth of the fluid layer are represented. The aspect ratios are: $(a)(3.2,1),(b)(4.8,2.7),(c)(4.0,1.5),(d)(3.2,2.5),(e)(4.8,3.2)$.

strip centred on $a_{1}=3.2$ describe, respectively three (figures $5 b$ and $5 e$ ) and two (figures $5 a$ and $5 d$ ) rolls parallel to $y$. There are also five rolls near $a_{1}=8.2$. The horizontal light grey zone with $a_{2}=1.6$ corresponds to a unique convective roll parallel to the $x$-axis (figure $5 c$ ), that is parallel to the larger sides of the box, and in the small light grey area near $\left(a_{1}, a_{2}\right)=(5.6,5.2)$, convection sets in in the form of three rolls parallel to the larger sides of the container.

In square containers, the convective structures are the following. For very small cavities $\left(a_{1} \leqslant 2.2\right)$, a degenerate solution is found in which one roll parallel to $y$ and one roll parallel to $x$ become simultaneously unstable at threshold. For $a_{1}=2.5$ ( $E E$ zone), a unique square cell is observed (figure $6 a$ ). For $a_{1}=4.4$, there is also a square cell (figure $6 b$ ) but the picture is somewhat different from figure 6(a). As a matter of fact, a cell similar to that corresponding to $a_{1}=2.6$, but rotated by $45^{\circ}$, is seen in the middle of figure $6(b)$ and the flow in the corners of the box is very weak. In connection with these pictures, it is interesting to wonder how square cells can be built in infinite layers. As recalled by Koschmieder (1993), the $(x, y)$-dependence of the vertical velocity in square cells can be written either as the sum $(w=(\cos k x$ $+\cos k y) W(z))$ or as the product $\left(w=\cos 2^{-1 / 2} k x \cos 2^{-1 / 2} k y W(z)\right)$ of the functions of $x$ and $y$ which define the vertical velocities corresponding to two sets of perpendicular rolls. Figure $6(a)$ is made up by the sum of two rolls perpendicular to $x$ and two rolls perpendicular to $y$ while figure $6(b)$ represents, rather, the product of such rolls. In both cases, the rolls parallel to both sides combine to give rise to a symmetric structure. For larger boxes (dark and light grey areas near $a_{1}=4.8$ ), the instability is degenerate and either three rolls perpendicular to $x$ or three rolls 
(a)

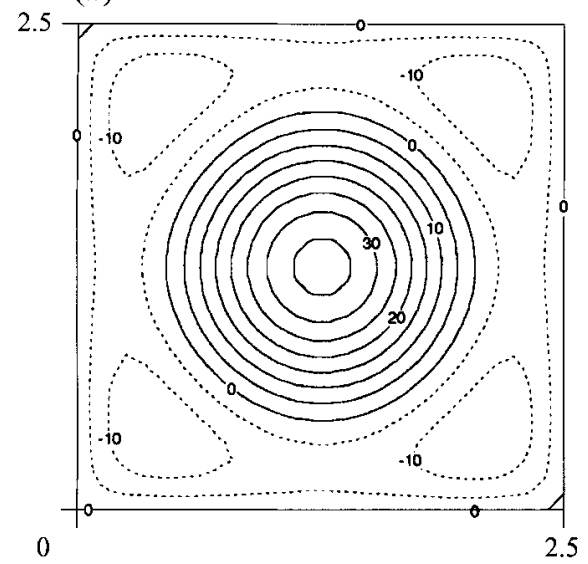

(c)

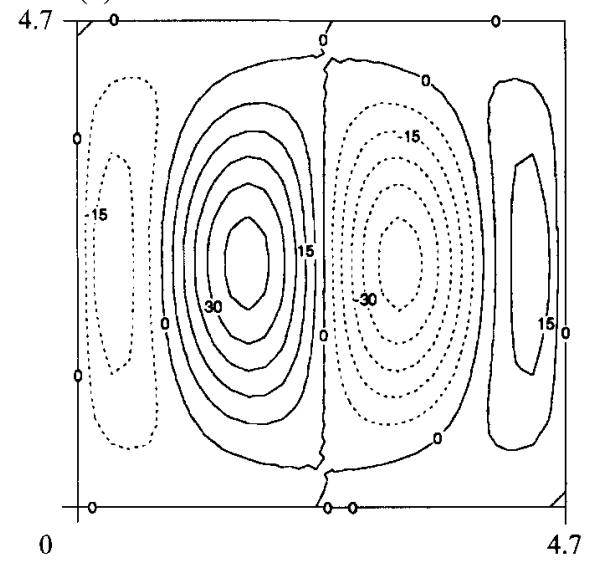

$(e)$

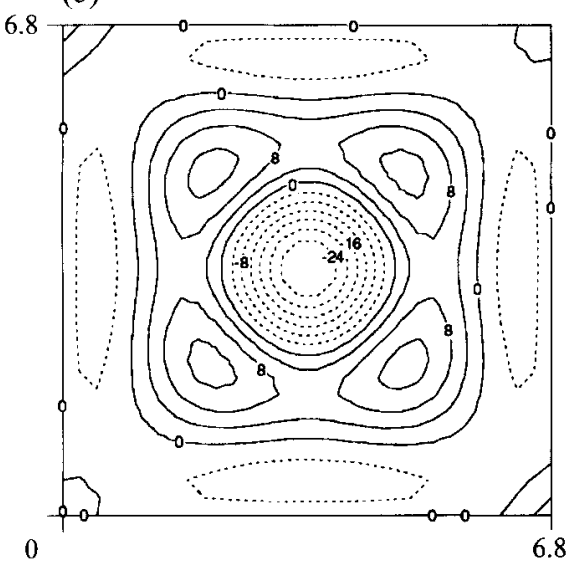

(b)

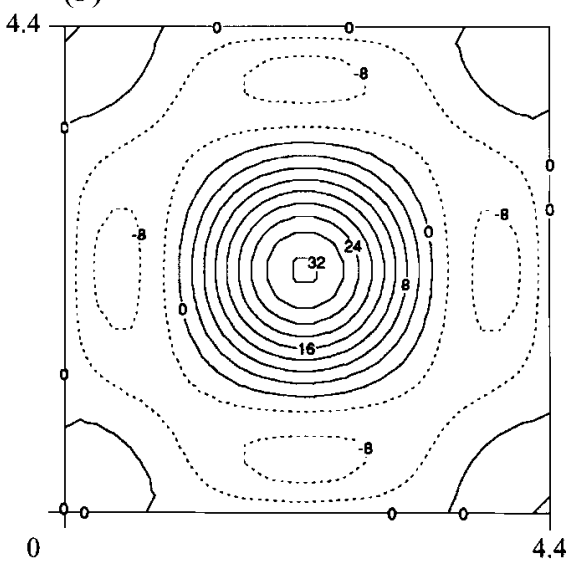

(d)

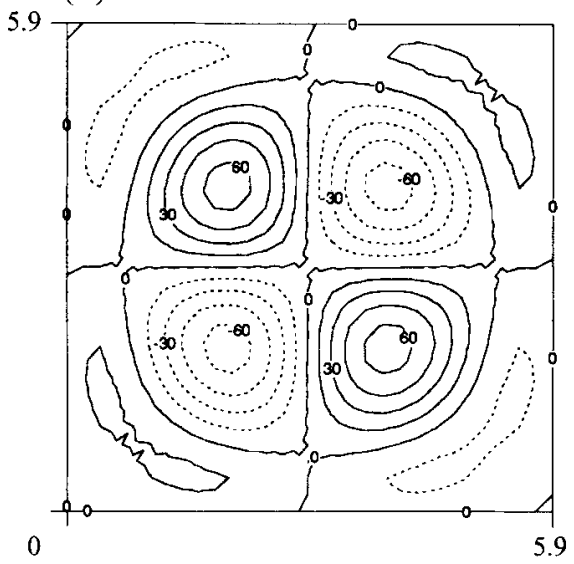

$(f)$

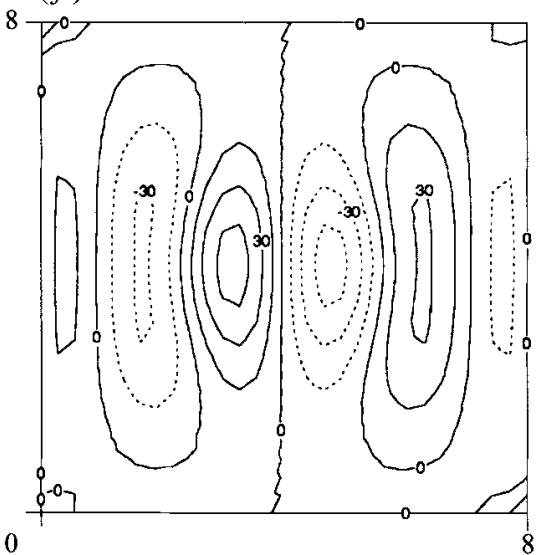

FIGURE 6. Representation of the flow at threshold for several square containers $(R a=B i=0)$. The iso-values of the vertical velocity $w$ at mid-depth of the fluid layer are represented. The aspect ratios are: (a) 2.5, (b) 4.4, (c) 4.7, (d) 5.9, (e) 6.8, (f) 8.0. 
perpendicular to $y$ appear (figure $6 c$ ). The pattern for the black $O O$ zone in the neighbourhood of $a_{1}=5.9$ is given in figure $6(d)$ and presents an 'antisymmetric' structure, which could be seen as the 'product' of three rolls parallel to $x$ and three rolls parallel to $y$. In the white area in the vicinity of $a_{1}=6.2$, the convective structure (figure $6 e$ ) consists of one square cell (similar to figure $6 a$, with opposite sign) in the middle of the box but a ring of sinking fluid is also observed along the sidewalls. For still larger values of the aspect ratio (light and dark grey zones near $a_{1}=8$ ), one observes five rolls parallel to any side of the container (figure $6 f$ ). For quasi-square containers, the convective cells are similar to these which appear in the square cavities but slight deformations are observed.

The degeneracy of the instability in square boxes may be related to the symmetry of the convective patterns. For $E E$ solutions (figures $6 a, 6 b$ and $6 e$ ), the convective structure is clearly invariant for a reflection about the medians of the box and also for a rotation of $90^{\circ}$ around the centre of the container. Similarly, the $O O$ solutions (figure $6 d$ ) have the same symmetry since the linear convective pattern is defined to within a change of sign. In both cases, the instability is non-degenerate and the critical eigenvalue is simple. On the other hand, when the eigenvector is an element of the $E O$ or $O E$ class, the structure is still invariant for the reflections with respect to the medians but the invariance is lost for a rotation of $90^{\circ}$ of the pattern. In fact, for square containers, the $E O$ or $O E$ modes are simultaneously unstable and the $E O$ pattern may be obtained by rotating the $O E$ one by $90^{\circ}$, and vice versa. In the light and dark grey zones of figure 2 , the instability is degenerate and the eigenvalue has an algebraic multiplicity equal to 2 .

A tentative physical interpretation of the geometrical nature of the convective cells at threshold in rectangular containers may be the following. The selection of the convective pattern is based on a balance between two arguments. The first one put forward by Davis (1967) and also taken up by Koschmieder (1993) states that, owing to dissipation at the rigid walls, a structure with rolls parallel to the shorter sides of the container dissipates less kinetic energy than a structure consisting of rolls parallel to the longer sides of the box. For this reason (Chandrasekhar 1961), the pattern with rolls parallel to the shorter sides is more likely to appear in experiments. The second argument which enables us to explain our results is the fact that the system prefers a structure for which the rolls are not compressed too much or dilated but have a dimension more or less equal to the dimension they would have in an infinite layer. For zero Biot and Rayleigh numbers, the critical wavenumber in infinite layers is about 2 and the width of the rolls is more or less equal to 1.6. Consider for instance a box with $\left(a_{1}, a_{2}\right)=(5,3.2)$. This box may be filled up with three rolls parallel to $y$ or with two rolls parallel to $x$. In both cases, the width of the rolls is about the 'optimal' value 1.6. The argument of dissipation along the walls will then impose the structure with three rolls parallel to the shorter sides of the container. In a box with $\left(a_{1}, a_{2}\right)=(5.7,5.2)$, three rolls parallel to the larger sides of the container can be observed because the rolls parallel to $y$ would not present an optimal width. All the results represented in figure 2 may be explained by using these two arguments. Of course, for large values of the aspect ratios, the argument about the width of the rolls becomes less relevant since the box can always be filled up with rolls of nearly optimal dimension. For this reason, the areas describing rolls parallel to the longer sides of the containers tend to disappear in large enough vessels. Let us also add that rolls parallel to each side may combine to form square or quasi-square cells in the neighbourhood of the diagonal $a_{1}=a_{2}$.

At this stage, it is interesting to compare our results with those of Dijkstra $(1995 a)$. Although both results are quite similar, our calculations are a bit more precise. For 
instance, in a square box with aspect ratio 4, Dijkstra gets the value 86.27 for the critical Marangoni number by using about $5 \times 16^{3} \approx 2 \times 10^{4}$ degrees of freedom (see Dijkstra's table 1). With $N_{x}=N_{y}=N_{z}=5$ (number of degrees of freedom = $\left.3 \times N_{x} \times N_{y} \times N_{z}=3 \times 5^{3}=375\right)$, we obtain $M a_{c}=85.771$. This value is highly reliable since for $\left(N_{x}, N_{y}, N_{z}\right)$ equal, respectively, to $(6,6,4),(7,7,5)$, and $(8,8,6)$, we found the values $85.718,85.771$ and 85.775 . This small lack of precision in Dijkstra's approach has no influence in small boxes where the successive eigenmodes bifurcate far from each other, but becomes important in larger boxes. For instance, Dijkstra missed the small $O O$ areas of figure 2 and the pattern presented in his figure $3(\mathrm{~g})$ does not correspond to the first linearly unstable eigenmode; the most unstable mode looks like our figure $6(d)$.

We would also like to mention that some patterns given by Dijkstra (1995a) are in fact not so different from one another as they may appear at first glance. For instance, his figures $3(d)-3(f)$ (boxes of aspect ratios 4.5, 5 and 5.5) correspond in our work to degenerate eigenmodes of classes $E O$ and $O E$ and the unique convective structure looks like our figure 6(c) (box of aspect ratio equal to 4.7; three convective rolls parallel to any side of the container). We think that Dijkstra's pictures look different from one another and also from our figure $6(c)$ because they are in fact some superposition of the two eigenmodes $E O$ and $O E$.

We now compare our work with the celebrated paper by Rosenblat et al. (1982b) who used the simplified boundary condition of slippery lateral walls. First of all, note that, with realistic no-slip lateral walls, the critical Marangoni number is systematically larger than the value 79.607 characterizing the instability in non-bounded domains. In the paper of Rosenblat et al. (1982b), this value 79.607 was found for many finite boxes, which is not physically realistic.

It is also interesting to compare our map of critical modes (figure 2) with the corresponding result of Rosenblat et al. (1982b) (see their figure 7). Although the two pictures look quite different, some interesting similarities emerge. Actually, the different strips corresponding to a fixed number of rolls also appear in the picture of Rosenblat et al., although if the borders of their 'strips' are more jagged. Indeed, the critical mode in containers with $a_{1} \approx \frac{1}{2} m \pi$ (or $a_{2} \approx \frac{1}{2} n \pi$ ) is always the mode $(m, 0)$ (or the mode $(0, n)$ ) and this mode consists of $m$ rolls parallel to $y$ (or $n$ rolls parallel to $x$ ). However, a difference exists between rigid and slippery walls because the strips corresponding to rolls parallel to the longer sides of the box do not disappear in large slippery boxes. This result is not surprising because, as in rigid boxes, the rolls always try to have an optimal width if the sidewalls are assumed slippery. However, in this case, no energy is dissipated along the walls and the rolls can therefore be found parallel to any border.

Another difference between both kinds of containers is that, in figure 7 of Rosenblat et al., some intermediate regions exist between the strips corresponding to pure rolls. Moreover, within each particular domain of their figure 7, the convective pattern is always self-similar and the only modification of the pattern within one domain is some dilation or contraction. In our figure 2, the different strips are contiguous and the convective pattern varies continuously within each strip.

The influence of non-zero Rayleigh and Biot numbers has also been considered in our analysis. In particular, the linear approach of the stability problem has been carried out for $R a=300, B i=0$ and for $R a=0, B i=10$. As expected, an increase of $R a$ corresponds to a decrease of the critical Marangoni number. Another relevant result is the fact that the map of the modes of convection obtained for a non-zero Rayleigh number is nearly completely similar to that of figure 2 . This means that gravity has 
nearly no influence on the selection of the linear convective pattern. In the case of infinite layers, a corresponding result is well known (Nield 1964; Lebon \& PérezGarcía 1980) since the critical wavenumber is nearly independent of the Rayleigh number. One has also observed that by increasing the Biot number, the critical Marangoni number grows. The map describing the convective patterns for $B i=10$ is also very similar to that for $B i=0$ (figure 2 ) but with the important restriction that the transitions between the different zones occur for smaller values of the aspect ratios: the map for $B i=10$ can be drawn by 'compressing' along both axes the map given in figure 2. This result may be related with the fact that, in infinite layers, the critical wavenumber decreases if $B i$ is increased (Nield 1964; Lebon \& Pérez-García 1980). A physical interpretation of this tendency was proposed by Nield (1964): when $B i$ is not zero, a larger temperature gradient is necessary to cause instability. More energy must be dissipated and smaller cells appear.

\section{Nonlinear Marangoni convection}

\subsection{The method of resolution}

To account for the evolution of the convective pattern above threshold, we follow a method first introduced by Eckhaus (1965). The same technique was used by Rosenblat et al. $(1982 a, b)$ in their nonlinear approach to Marangoni convection in slippery boxes and by Parmentier et al. (1996) in the case of horizontally infinite boxes. Some general considerations on this nonlinear approach to convection may be found, for instance, in Manneville (1990). Note also that the adjoint linear eigenvalue problem is required to apply the method. For completeness, this adjoint problem is given in Appendix B.

The method consists first in expanding the solution of the nonlinear equations in series of the eigenmodes of the linear problem. The time-dependent coefficients are the so-called ' amplitudes' of the different modes of convection. The eigenmodes to be used here are the eigenmodes of set (3.1)-(3.3) considered as an eigenvalue problem for the growth rate $\sigma$, when the Marangoni number is fixed and equal to $M a_{c}$. Clearly the smallest eigenvalue of this problem is zero and corresponds to the marginally stable convective mode. If the eigenmodes are assumed to form a complete set, one may write:

$$
\left(\begin{array}{l}
v \\
\theta
\end{array}\right)=\sum_{p} A_{p}(t)\left(\begin{array}{l}
v_{p} \\
\theta_{p}
\end{array}\right),
$$

where $A_{p}(t)$ are the amplitudes and $\left(v_{p}, \theta_{p}\right)$ denote the eigenmodes (of any parity $E E$, $E O, O E$ or $O O$ ) of the linear eigenvalue problem for $\sigma$ with $M a=M a_{c}$. All the boundary conditions, except the Marangoni conditions at the upper surface, are automatically fulfilled if the unknown fields take the form (4.1). Moreover, the continuity equation (2.1) is also directly satisfied. The Marangoni conditions can, however, be taken into account by slightly modifying expression (4.1): indeed, the continuity equation and all the boundary conditions are satisfied by:

$$
\left(\begin{array}{l}
\boldsymbol{v} \\
\theta
\end{array}\right)=\sum_{p} A_{p}(t)\left(\begin{array}{c}
\boldsymbol{v}_{p} \\
\frac{M a_{c}}{M a} \theta_{p}
\end{array}\right) .
$$

Note that, for the forthcoming developments, it is worth assuming that the eigenmodes are ordered by decreasing growth rate $\sigma_{p}: \sigma_{p} \leqslant \sigma_{q} \leqslant 0$ if $p>q$.

Introduce then this development (4.2) in the nonlinear equations (2.2)-(2.3) for the velocity and temperature. Multiply (2.2) by $v_{p}^{*}$ and (2.3) by $\left(M a / M a_{c}\right) \theta_{p}^{*}$, where 
$\left(v_{p}^{*}, \theta_{p}^{*}\right)$ denotes an eigenmode, with eigenvalue $\sigma_{p}$, of the adjoint eigenvalue problem (B 1)-(B 8) considered as an eigenvalue problem for the growth rate when $M a=M a_{c}$. Add both expressions and integrate over the fluid volume. After performing integrations by parts and using the boundary conditions and the bi-orthogonality relations (B 9), one obtains the following evolution equations for the amplitudes $A_{p}(t)$ :

$$
\frac{\mathrm{d} A_{p}}{\mathrm{~d} t}=\sigma_{p} A_{p}+\epsilon \sum_{q} T_{0}(p, q) A_{q}+\sum_{q, l} T_{1}(p, q, l) A_{q} A_{l} .
$$

In this expression, $\epsilon$ is the relative distance to threshold. It is defined as

$$
\epsilon=\frac{M a-M a_{c}}{M a_{c}} \text {. }
$$

The matrices in (4.3) are given by

$$
\begin{gathered}
T_{0}(p, q)=\frac{\left\langle\theta_{p}^{*} w_{q}\right\rangle}{\left\langle\theta_{p}^{*} \theta_{p}\right\rangle+\operatorname{Pr}^{-1}\left\langle\boldsymbol{v}_{p}^{*} \cdot \boldsymbol{v}_{p}\right\rangle}, \\
T_{1}(p, q, l)=-\frac{\left\langle\theta_{p}^{*}\left(\boldsymbol{v}_{q} \cdot \nabla \theta_{l}\right)+\operatorname{Pr}^{-1} \boldsymbol{v}_{p}^{*} \cdot\left\{\left(\boldsymbol{v}_{q} \cdot \nabla\right) \boldsymbol{v}_{l}\right\}\right\rangle}{\left\langle\theta_{p}^{*} \theta_{p}\right\rangle+\operatorname{Pr}^{-1}\left\langle\boldsymbol{v}_{p}^{*} \cdot \boldsymbol{v}_{p}\right\rangle} .
\end{gathered}
$$

The set (4.3) forms an infinite dimensional system of nonlinear ordinary differential equations for the amplitudes of the eigenmodes and is the basis of our nonlinear analysis.

\subsection{Reduction of the dynamics in the weakly nonlinear regime}

To be tractable, the infinite dimensional set (4.3) must be simplified and, in particular, it must be restricted to a finite number of equations. The use of a finite number of equations can be justified by noting that, in the weakly nonlinear regime, only a small number of eigenmodes are linearly unstable while all other modes have negative growth rates. It is convenient to share out the eigenmodes into two sets. The first set contains the 'unstable' eigenmodes which bifurcate in the vicinity of the threshold and which are thus actually unstable in the neighbourhood of $\epsilon=0$. The $\epsilon$-value defining the bifurcation of the successive eigenmodes is easily obtained from (4.3). It is given by

$$
\epsilon_{p}=-\frac{\sigma_{p}}{T_{0}(p, p)}
$$

where $\epsilon_{p}$ denotes the value of $\epsilon$ at which the eigenmode $p$ bifurcates. The 'unstable' eigenmodes are defined by an index running from 1 to some number $N_{U}$ which must be determined.

The second set of eigenmodes is made up by the 'stable' eigenmodes whose linear growth rate remains negative in the weakly nonlinear regime. In the weakly nonlinear regime, this growth rate is given by

$$
\sigma_{p}^{*}=\sigma_{p}+\epsilon T_{0}(p, p)
$$

These stable eigenmodes do not really participate in the dynamics of the system but they cannot be omitted since they represent the nonlinear response of the system to the growth of the unstable modes. These modes are generated by the quadratic interactions of the first unstable modes and their amplitude should be expressed as a quadratic expression in the amplitudes of the unstable modes. To be more explicit, equations (4.3) for the stable modes will be simplified in the following way. First, the term proportional to $\epsilon$ in (4.3) may be disregarded with respect to $\sigma_{p} A_{p}$ since $\epsilon$ remains small in the weakly nonlinear regime and $\sigma_{p}$ is quite negative for a well-damped 
eigenmode. Secondly, the time derivative of the amplitude is neglected since the stable modes do not participate in the dynamics of the system. Finally, the quadratic term may be restricted to a quadratic expression containing only the amplitudes of the unstable modes because, in the neighbourhood of threshold, the amplitudes of the unstable modes remain rather small and terms of order higher than 2 in these amplitudes may be neglected. The amplitudes of the stable modes can thus be written as:

$$
A_{s_{i}}=-\frac{1}{\sigma_{s_{i}}} \sum_{u_{j} u_{k}(u n s t a b l e)} T_{1}\left(s_{i}, u_{j}, u_{k}\right) A_{u_{j}} A_{u_{k}} .
$$

In (4.9), and subsequently, indices $s_{i}$ denote stable modes while indices $u_{i}$ refer to unstable eigenmodes.

Formula (4.9) shows also that the amplitudes $A_{s_{i}}$ become smaller and smaller as index $s_{i}$ increases since the corresponding growth rates become more and more negative. In particular, eigenmodes with sufficiently negative growth rate may be disregarded since their amplitude becomes negligibly small. In practice, the contributions of the stable modes will only be taken into account for $s_{i}$ larger than $N_{U}$ but smaller than $N_{U}+N_{S}$, for some integer $N_{S}$ which must also be evaluated.

By introducing (4.9) with $N_{U}<s_{i} \leqslant N_{U}+N_{S}$ in (4.3), we obtain the final equations:

$$
\begin{aligned}
\frac{\mathrm{d} A_{u_{i}}}{\mathrm{~d} t}= & \sigma_{u_{i}} A_{u_{i}}+\epsilon \sum_{u_{j}(u n s t)} T_{0}\left(u_{i}, u_{j}\right) A_{u_{j}} \\
& +\sum_{u_{j}, u_{k}(u n s t)} T_{1}\left(u_{i}, u_{j}, u_{k}\right) A_{u_{j}} A_{u_{k}}+\sum_{u_{j}, u_{k}, u_{l}(u n s t)} T_{2}\left(u_{i}, u_{j}, u_{k}, u_{l}\right) A_{u_{j}} A_{u_{k}} A_{u_{l}},
\end{aligned}
$$

with $u_{i}=1, \ldots, N_{U}$. Note that, in (4.10), we have neglected terms proportional to $\epsilon A_{u_{i}} A_{u_{j}}$ and terms of order higher than 3 in the amplitudes of the unstable modes. The matrix $T_{2}$ is given by:

$$
T_{2}\left(u_{i}, u_{j}, u_{k}, u_{l}\right)=\sum_{s_{m}(s t)}\left(-\frac{1}{\sigma_{s_{m}}}\right)\left(T_{1}\left(u_{i}, u_{j}, s_{m}\right)+T_{1}\left(u_{i}, s_{m}, u_{j}\right)\right) T_{1}\left(s_{m}, u_{k}, u_{l}\right) .
$$

The above reduction of the dynamics of the system to a finite number of ordinary differential equations for the most unstable modes is the well-known adiabatic elimination of the slaved modes. The procedure has been widely developed in earlier works (see, for instance, Manneville 1990; Haken 1983; Rosenblat et al. $1982 a, b$; Foias et al. 1988) and will not be discussed further. It will now be applied to the study of Marangoni convection in no-slip rectangular vessels. Note that the values of the (numerous) coefficients $T_{0}, T_{1}$ and $T_{2}$ will not be given explicitly in the paper. However, these numbers could be provided on request to interested parties.

\subsection{Nonlinear Marangoni convection in rigid containers}

The application of the above method of reduction requires first the determination of the numbers $N_{U}$ and $N_{S}$ of the unstable and stable modes.

First, let us stress that we have basically in mind the determination of the bifurcation diagrams in the weakly nonlinear regime. Therefore, $N_{U}$ and $N_{S}$ will be selected in order to provide a good description of the stable (i.e. observable) branches of the bifurcation diagrams in a range of $\epsilon$-values less than about $15 \%$. For these values of 


\begin{tabular}{ccccc}
\hline & $\epsilon_{u}=15 \%$ & $\epsilon_{u}=20 \%$ & $\epsilon_{u}=25 \%$ & $\epsilon_{u}=25 \%$ \\
& $\sigma_{s}=-10$ & $\sigma_{s}=-10$ & $\sigma_{s}=-12$ & $\sigma_{s}=-16$ \\
& $\left(N_{U}=2+2+2+1\right.$ & $\left(N_{U}=4+2+2+2\right.$ & $\left(N_{U}=5+2+2+2\right.$ & $\left(N_{U}=5+2+2+2\right.$ \\
& $\left.N_{S}=9+7+7+7\right)$ & $\left.N_{S}=7+7+7+6\right)$ & $\left.N_{S}=8+8+8+6\right)$ & $\left.N_{S}=8+10+10+9\right)$ \\
$\epsilon(t p)$ & 0.0165 & 0.0165 & 0.0166 & 0.0166 \\
$\epsilon\left(b_{1}\right)$ & 0.0210 & 0.0215 & 0.0204 & 0.0203 \\
$\epsilon\left(b_{2}\right)$ & 0.0274 & 0.0250 & 0.0254 & 0.0251 \\
$\epsilon\left(b_{3}\right)$ & 0.111 & 0.102 & 0.102 & 0.103
\end{tabular}

TABLE 4. Positions of $t p, b_{1}, b_{2}$ and $b_{3}$ in the bifurcation diagram corresponding to $\left(a_{1}, a_{2}\right)=(4.7,4.6)$ (see figure 8) as functions of $\epsilon_{u}$ and $\sigma_{s}$; these parameters are defined by the fact that the 'unstable' modes bifurcate for $\epsilon<\epsilon_{u}$ and that the 'stable' modes taken into account have a growth rate $\sigma>\sigma_{s}$. The values of $N_{U}$ and $N_{S}$ are written as sums of four numbers which give the numbers of eigenmodes of parity $E E, E O, O E$ and $O O$.

the relative distance to threshold and for $B i=0, \operatorname{Pr}=10^{4}$, we have checked that the stable branches of the bifurcation diagrams were properly described by choosing the stable and unstable eigenmodes in the following way. First, the unstable modes are these which bifurcate for $\epsilon$-values less than $25 \%$ (and also those modes whose growth rates are less negative than the growth rates of the modes which bifurcate before $\epsilon=25 \%$; an example of such a mode is the mode for which the velocity vanishes and the temperature is a function of $z$ only: this mode never bifurcates and its growth rate is equal to $-\frac{1}{4} \pi^{2}$ (Parmentier et al. 1996)). Secondly, the stable modes are the modes with index higher than $N_{U}$ and growth rate higher than the value -12 . Of course the values of $N_{U}$ and $N_{S}$ determined using these conditions depend on the aspect ratios of the container. It is also worth stressing that the eigenmodes with growth rates larger than -12 are correctly approximated by (3.4) for boxes with aspect ratios less than about 7 if the number of trial functions is fixed by $\left(N_{x}, N_{y}, N_{z}\right)=(7,7,5)$. Note also that, before considering three-dimensional situations, we have checked the validity of the method by comparing with the results obtained by Dijkstra (1992) for twodimensional containers: the agreement was found to be very good. For threedimensional containers, the convergence of the procedure when $N_{U}$ and $N_{S}$ are increased has been checked in the following way. First of all, we have searched for a qualitative convergence of the bifurcation diagram, that is, we have checked that the number and the kind $(E E, E O, O E$ or $O O)$ of the stable branches appearing for $\epsilon$ less than about $15 \%$ were not changed if $N_{U}$ and/or $N_{S}$ were increased. Secondly, quantitative information about the convergence may also be obtained by computing the positions of some secondary bifurcation points for different values of $N_{U}$ and $N_{S}$. The results for a box of aspect ratio $\left(a_{1}, a_{2}\right)=(4.7,4.6)$ are reported in table 4 . The positions of the turning point $t p$ and the secondary bifurcation points $b_{1}, b_{2}$ and $b_{3}$ (see figure 8) are given for different values of $N_{U}$ and $N_{S}$. The table shows that choosing the unstable and stable modes as explained above gives a good approximation of the bifurcation diagram.

We now examine the results corresponding to a representative sample of boxes with aspect ratios smaller than 8 . The selected boxes are indicated with crosses in figure 2 . The Biot number is fixed to 0 and the Prandtl number is equal to $10^{4}$. In order to avoid the degeneracy of the $E O$ and $O E$ modes in square geometries we study in the first subsection the case of quasi-square containers. These quasi-square containers are also more realistic from an experimental point of view since a perfectly square geometry can never be achieved in practice. 

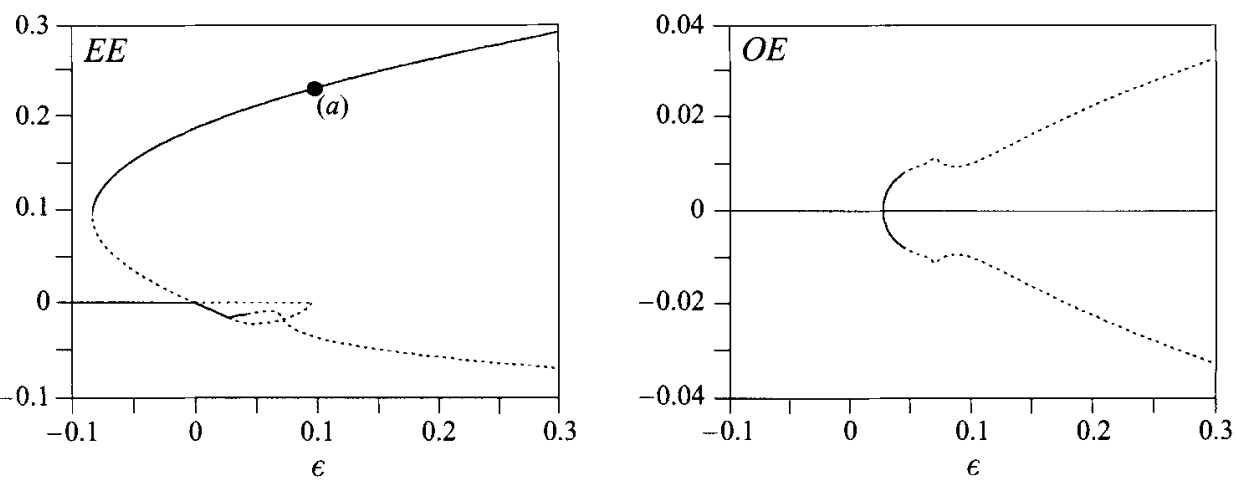

FigURF 7. Bifurcation diagram for a quasi-square box of aspect ratios $\left(a_{1}, a_{2}\right)=(2.5,2.4)$.

\subsubsection{Quasi-square containers}

(a) Quasi-square container with aspect ratios $\left(a_{1}, a_{2}\right)=(2.5,2.4)$

The critical Marangoni number for $\left(a_{1}, a_{2}\right)=(2.5,2.4)$ is given by $M a_{c}=106.07$ and the critical solution belongs to the $E E$ class. In such a box, two modes with parity $E E$, one $E O$-mode, one $O E$-mode and one $O O$-mode bifurcate before $\epsilon=25 \%$, so that $N_{U}$ is equal to 5 . For conciseness and to recall the repartition between the four parity classes, we will write $N_{U}=2+1+1+1$. With the same notation, one has $N_{S}=2+2+2+2$. The final system describing the weakly nonlinear regime consists thus in five cubic o.d.e. for the amplitudes of the five unstable modes. This set of equations will not be written explicitly but we shall determine the corresponding bifurcation diagram. The latter is obtained by using standard continuation techniques as described for instance in Seydel (1988). A complete bifurcation diagram should give the amplitudes of all the unstable modes as functions of the distance to the threshold. However, it is sufficient for our discussion to represent only the amplitude of the most unstable mode of each parity. Moreover, it would not be useful to represent all the unstable branches since they would make the pictures very intricate and also because these branches correspond to solutions which are not experimentally observable. In the graphs, we will use solid curves to represent stable solutions and dashed lines for unstable ones. The diagrams are also extrapolated to values of $\epsilon$ a bit larger than $15 \%$. Note, finally, that if some amplitude remains always equal to zero, its representation as a function of $\epsilon$ is not given.

The bifurcation diagram for a box of aspect ratios $\left(a_{1}, a_{2}\right)=(2.5,2.4)$ is given in figure 7. At the threshold, a transcritical bifurcation towards a solution made up by $E E$ modes only can be observed. The width of the subcritical domain is equal to about $8.3 \%$. At $\epsilon=2.7 \%$, there is a secondary bifurcation of the $O E$ mode. In a progressive heating experiment, the real finite-amplitude perturbations make the system jump on the stable positive $E E$ branch before reaching the threshold. For this solution, the motion sets in taking the form of a square cell in which the fluid rises at the centre. The convective pattern corresponding to point $(a)$ in the bifurcation diagram is represented in figure $12(a)$. The structure corresponding to negative values of the $E E$ amplitude and non-zero $O E$ mode is not drawn because, in a progressive heating experiment, only the positive $E E$ branch will be observed and also because the range of $\epsilon$ for which these solutions are stable is rather small.

In the same $E E$ area of figure 2, we have also considered the box with $\left(a_{1}, a_{2}\right)=(4.2$, 4.1). We found a bifurcation diagram quite similar to that given in figure 7 . However, 

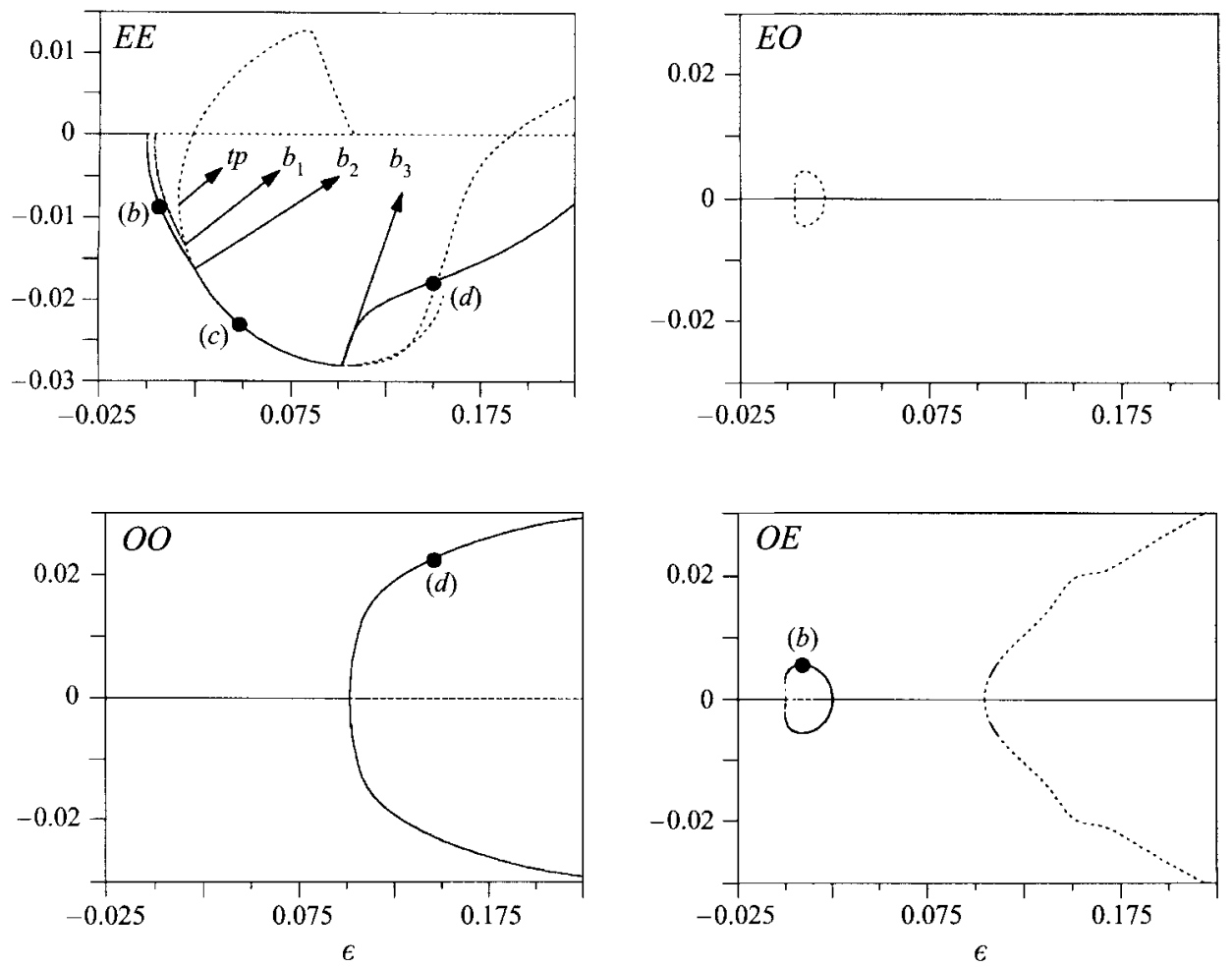

FIGURE 8. Bifurcation diagram for a quasi-square box of aspect ratios $\left(a_{1}, a_{2}\right)=(4.7,4.6)$.

the convective pattern observed in a progressive heating experiment is different since one observes a unique square cell which is rotated by $45 \%$ with respect to the cell given in figure 12(a). This kind of convective pattern also appears in the box described hereinafter and a picture of the motion in this box is given in figure $12(c)$.

(b) Quasi-square container with aspect ratios $\left(a_{1}, a_{2}\right)=(4.7,4.6)$

In the present example, the instability is nearly degenerate. The $O E$ mode bifurcates first but the $E O$ solution becomes unstable quite near the threshold. The numbers of unstable and stable modes are $N_{U}=5+2+2+2$ and $N_{S}=8+8+8+6$. The bifurcation diagram is given in figure 8 . At the threshold, one has a subcritical bifurcation towards the $O E$ mode but the subcritical domain is very small. The $E E$ modes are non-zero for this solution, since they are generated by the quadratic selfinteraction of the $O E$ mode. The pattern corresponding to this branch (point $b$ : $\epsilon=1.8 \%$ ) is represented in figure $12(b)$. One main cell appears which is not located at the centre of the container. Two other small areas of weak upflow also appear in the left-hand corners of the box. This picture corresponds to a positive value of the amplitude of the first $O E$ mode. A negative value of the $O E$-amplitude would produce a pattern which is the reflection of figure $12(b)$ with respect to $x=\frac{1}{2} a_{1}$. It is important to note that this convective structure is quite different from that found from the linear approach (see figure $6 c$ ). This is due to the presence of the $E E$ modes which are generated by the nonlinear evolution of the $O E$ mode. The branch corresponding to this solutions ends up at a secondary bifurcation point on the branch originating from the bifurcation of the $E E$ solution. At this point, the $E E$ solution becomes stable and the observable pattern takes the form of a unique square cell with upflow in the centre. 

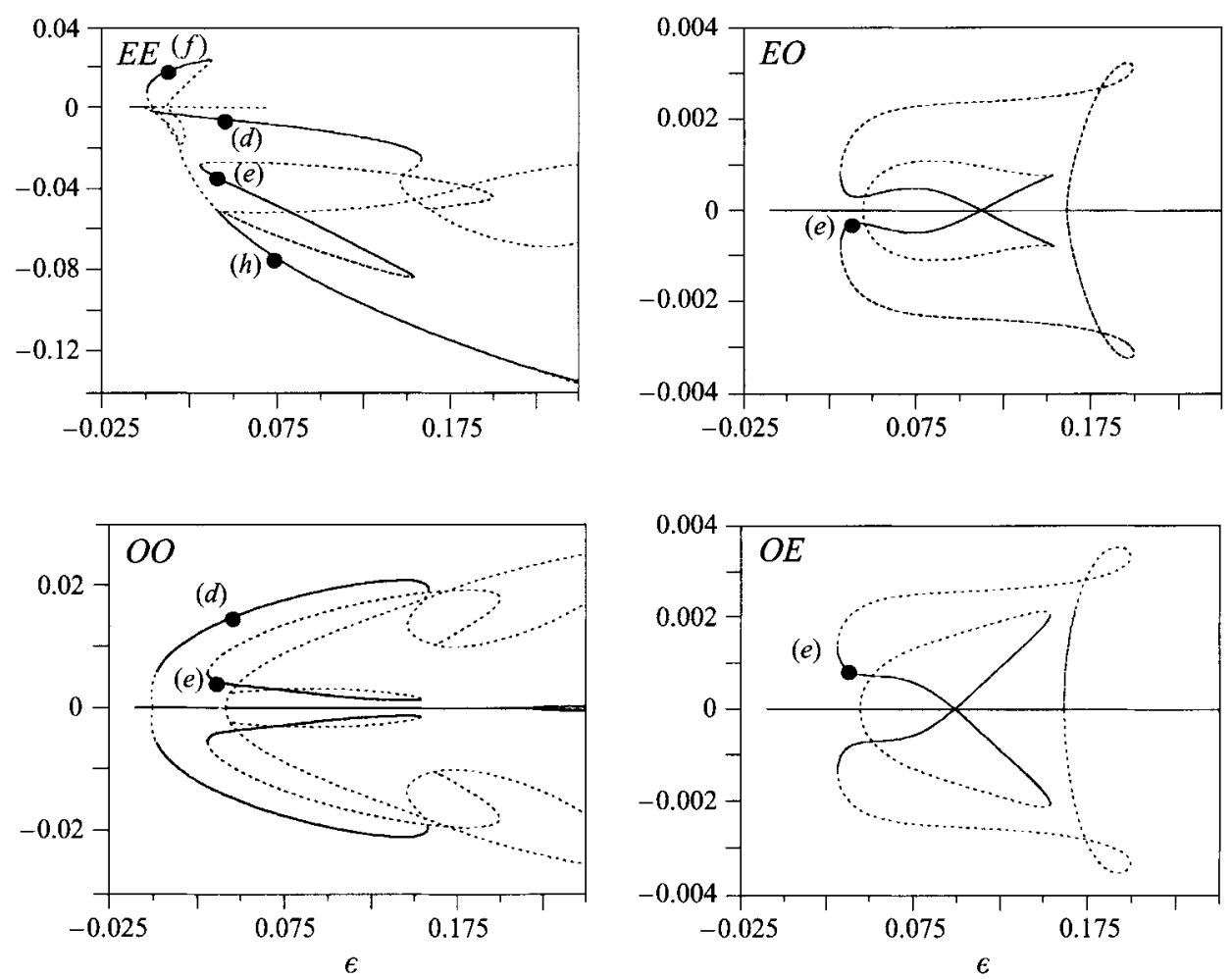

FIGURE 9. Bifurcation diagram for a quasi-square box of aspect ratios $\left(a_{1}, a_{2}\right)=(5.94,5.9)$.

This cell is represented in figure $12(c)$ for point $(c)$ in the diagram. For larger values of $\epsilon$, one observes another secondary bifurcation towards a solution made up by the superposition of the $E E$ and $O O$ modes (point $(d)$ ). On this branch, the convective pattern consists of two triangular cells with upflow at the centre of each cell. A picture of the structure is given in figure $12(d)$. This picture corresponds in fact to a box of aspect ratios $\left(a_{1}, a_{2}\right)=(5.94,5.95)$ in which such triangular cells also appear. In the bifurcation diagram, we have represented the (unstable) branch which arises at the bifurcation of the $E O$ mode in order to show the quasi-degeneracy of the instability.

We have also studied a slightly larger container in the same $E O-O E$ domain. For $\left(a_{1}, a_{2}\right)=(5.4,5.3)$, the bifurcation diagram presents the following general features. At threshold, the $E O$ mode bifurcates subcritically to give a stable structure similar to figure $12(b)$ but rotated by $90^{\circ}$. The $O E$ mode bifurcates also subcritically quite near the threshold and gives rise to a pattern similar to figure $12(b)$. These $E O$ or $O E$ solutions do, however, not exist for large value of $\epsilon$. For a distance to the threshold in the neighbourhood of $10 \%$, a four-square-cells solution, a two-triangular-cells pattern and a three-convective-cells structure coexist (figures $12 d-12 f$ ). These three kinds of convective structures are also present in larger boxes and will be discussed in more detail later on.

(c) Quasi-square container with aspect ratios $\left(a_{1}, a_{2}\right)=(5.94,5.9)$

In figure 2 , the point $\left(a_{1}, a_{2}\right)=(5.94,5.9)$ is in a black area where the $O O$ mode becomes first unstable. The bifurcation diagram for this container is given in figure 9 $\left(N_{U}=7+5+5+5\right.$ and $\left.N_{S}=12+11+11+8\right)$. The bifurcation at threshold is subcritical with a very narrow domain of (unstable) subcritical convection. The $E E$ 

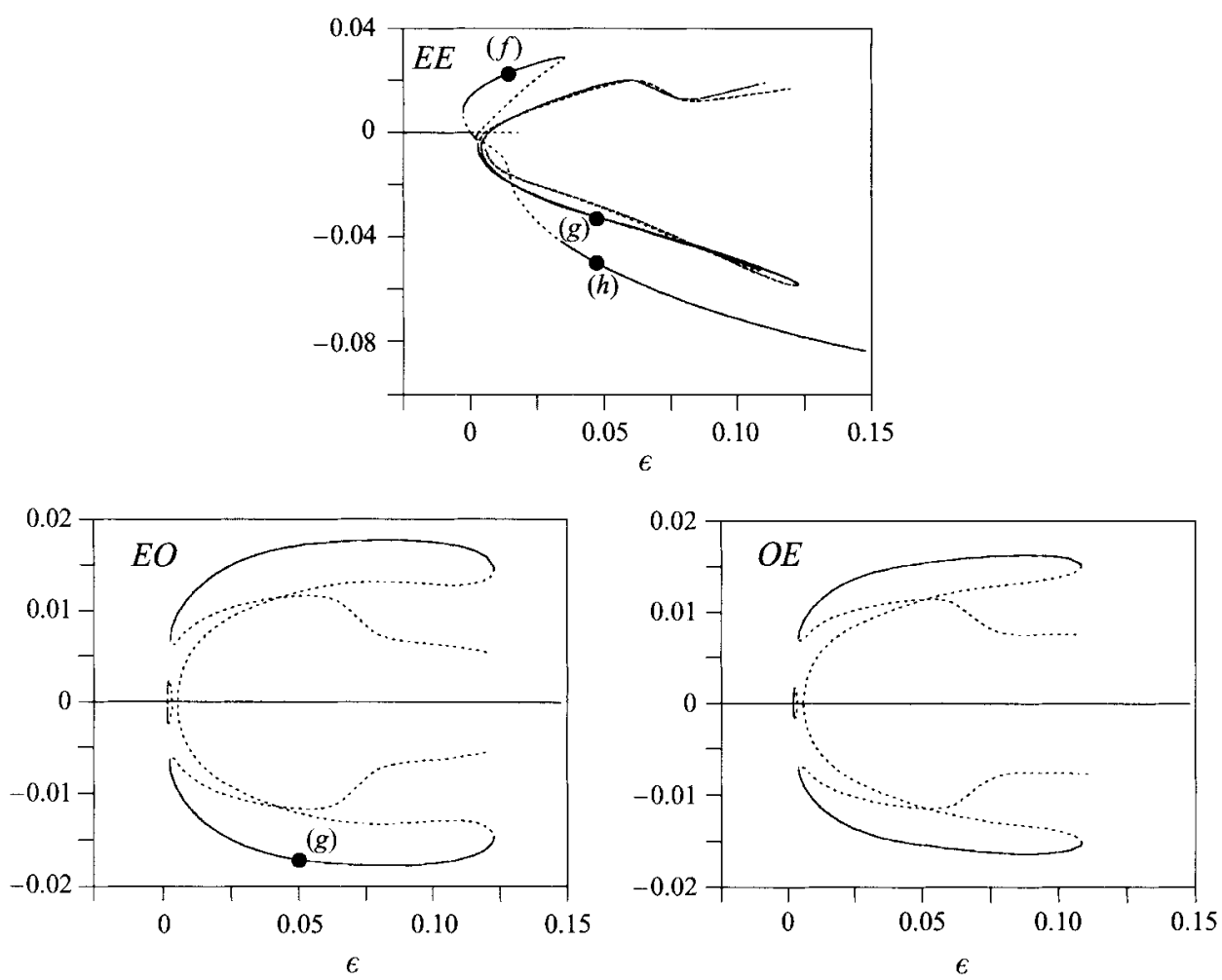

FIGURE 10. Bifurcation diagram for a quasi-square box of aspect ratios $\left(a_{1}, a_{2}\right)=(6.9,6.8)$.

mode bifurcates very near threshold and, actually, the only non-conductive solution at $\epsilon=0$ is the $E E$ mode. This branch (point $f$ ) corresponds to a square cell with upflow at the centre and a narrow band of rising fluid along the sidewalls (see figure $12(f)$ for a similar convective pattern). The $O O$ branch originating at the threshold becomes stable for a positive but very small value of $\epsilon$. The convective pattern for point $(d)$ on this branch is given in figure $12(d)$ and consists of two triangular cells with upflow at the centre of each cell. This structure results from the superposition of the unstable $O O$ mode and the $E E$ and $O O$ modes generated in the nonlinear regime. Figure $12(d)$ corresponds to a positive value of the amplitude of the first $O O$ mode. A negative value for this amplitude would give a similar structure but rotated by $90^{\circ}$. Consider now the 'negative part' of the $E E$ branch. This branch becomes stable for $\epsilon=4.2 \%$. The convective pattern for this stable branch is made up by four square cells similar to the configuration observed in figure $12(a)$. The pattern for point $H$ is similar to that given in figure $12(h)$ which corresponds to a square box of aspect ratio 6.8. Another stable branch is also present in the bifurcation diagram. On this branch, modes of all parities are non-zero and the structure is the superposition of $E E, E O, O E$ and $O O$ modes. In figure $12(e)$ is plotted the convective structure corresponding to point $(e)$ in the bifurcation diagram. Three convective cells are clearly distinguished with one square cell in the lower left-hand corner and two other wedge-shaped cells. Of course, the square cell could also be found in other corners (the $O O$ amplitude may take both signs and, for each sign, the $E O$ and $O E$ amplitudes may also be positive or negative). 

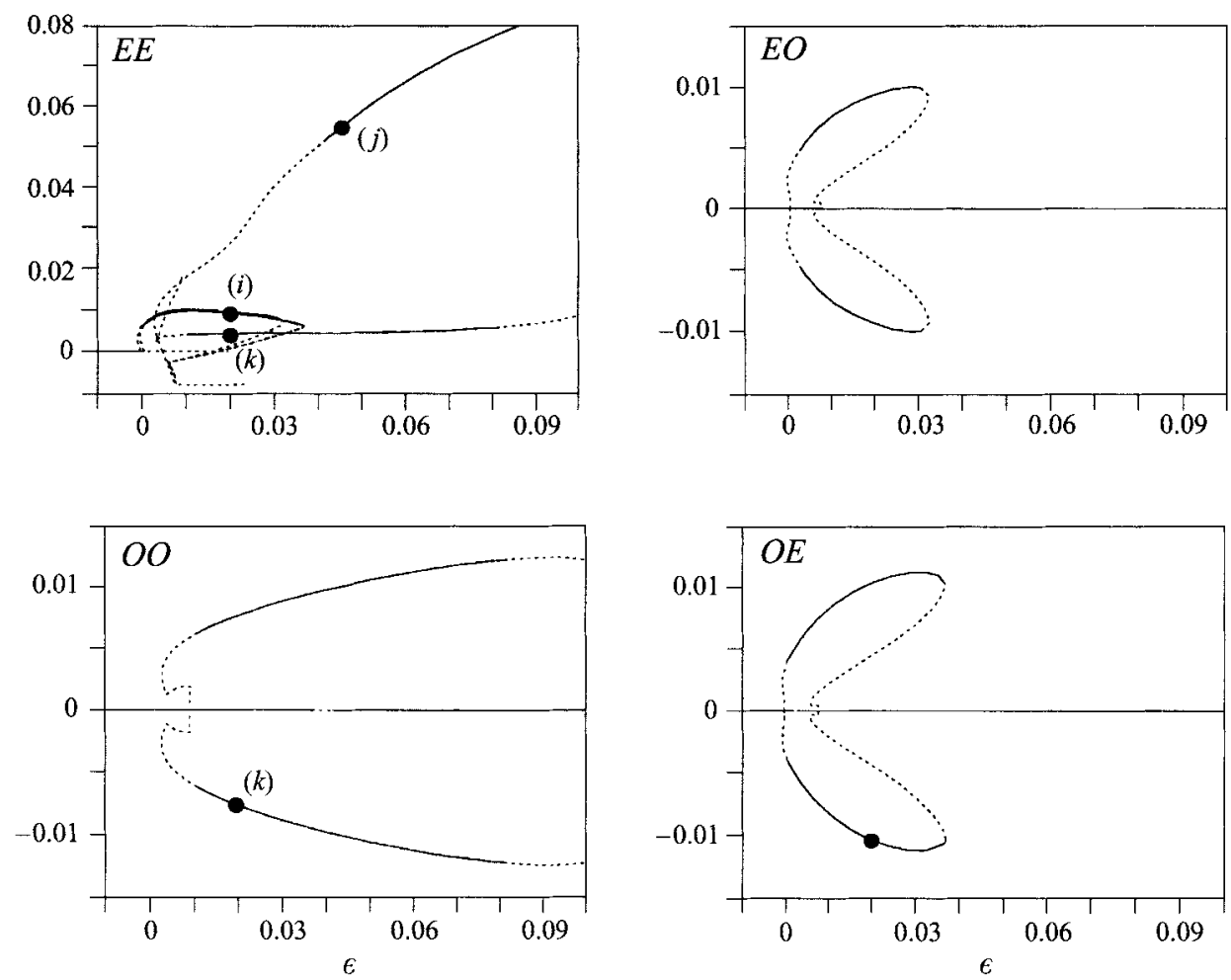

FIGURE 11. Bifurcation diagram for a quasi-square box of aspect ratios $\left(a_{1}, a_{2}\right)=(8.1,8)$.

(d) Quasi-square container with aspect ratios $\left(a_{1}, a_{2}\right)=(6.9,6.8)$

The critical eigenmode has parity $E E$ for $\left(a_{1}, a_{2}\right)=(6.9,6.8)$. The numbers of eigenmodes to take into account are $N_{U}=9+8+8+6$ and $N_{S}=13+13+13+13$. The bifurcation diagram is plotted in figure 10. The bifurcation at threshold is transcritical with a $0.29 \%$ width subcritical domain. Near $\epsilon=0$, the negative part of the first $E E$ branch is stable but soon becomes unstable owing to the secondary bifurcations of the $E O$ and $O E$ modes. These stable solutions exist only in very narrow windows of $\epsilon$ and could thus not easily be observed. Therefore, the convective structure is not represented. When the fluid layer is progressively heated, the system jumps onto the stable positive $E E$ branch before reaching threshold. On this branch, the convective pattern for point $(f)$ takes the form given by figure $12(f)$ : a square cell with upflow at the centre and a narrow band of rising fluid along the sidewalls. This solution becomes unstable for $\epsilon=3.5 \%$ and the system must jump onto another branch. For $\epsilon>3.4 \%$, the negative $E E$ branch becomes stable and the convection takes the form of four square cells (see figure $12(h)$ for point $h$ ). Note also that this branch is the only stable solution for sufficiently large values of $\epsilon$. Other stable solutions are also possible. These take the form of either a superposition of $E O$ and $E E$ modes or a superposition of $O E$ and $E E$ modes. The pattern corresponding to point $(g)$ in the bifurcation diagram consists of a three-cell solution as shown in figure $12(\mathrm{~g})$. The three cells are, however, differently arranged to those in figure $12(e)$. The pattern corresponding to a positive value of the $E O$ mode can be obtained by a reflection of figure $12(\mathrm{~g})$ about $y=\frac{1}{2} a_{2}$. Similarly, the $O E-E E$ solutions are obtained by rotating by $90^{\circ}$ in both directions the pattern of figure $12(\mathrm{~g})$. The coexistence of nearly equivalent solutions for 

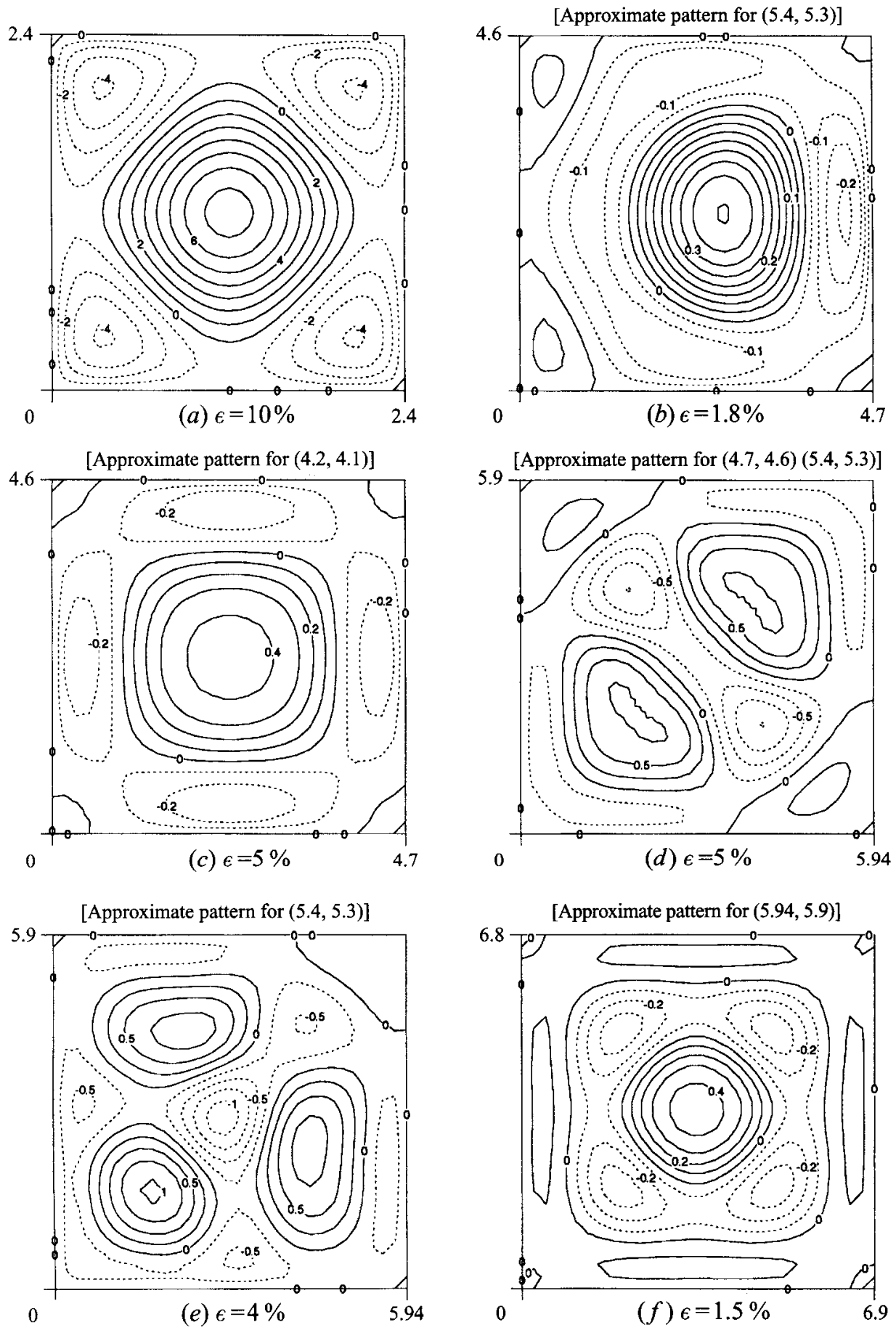

FIGURE $12(a-f)$. For caption see facing page. 

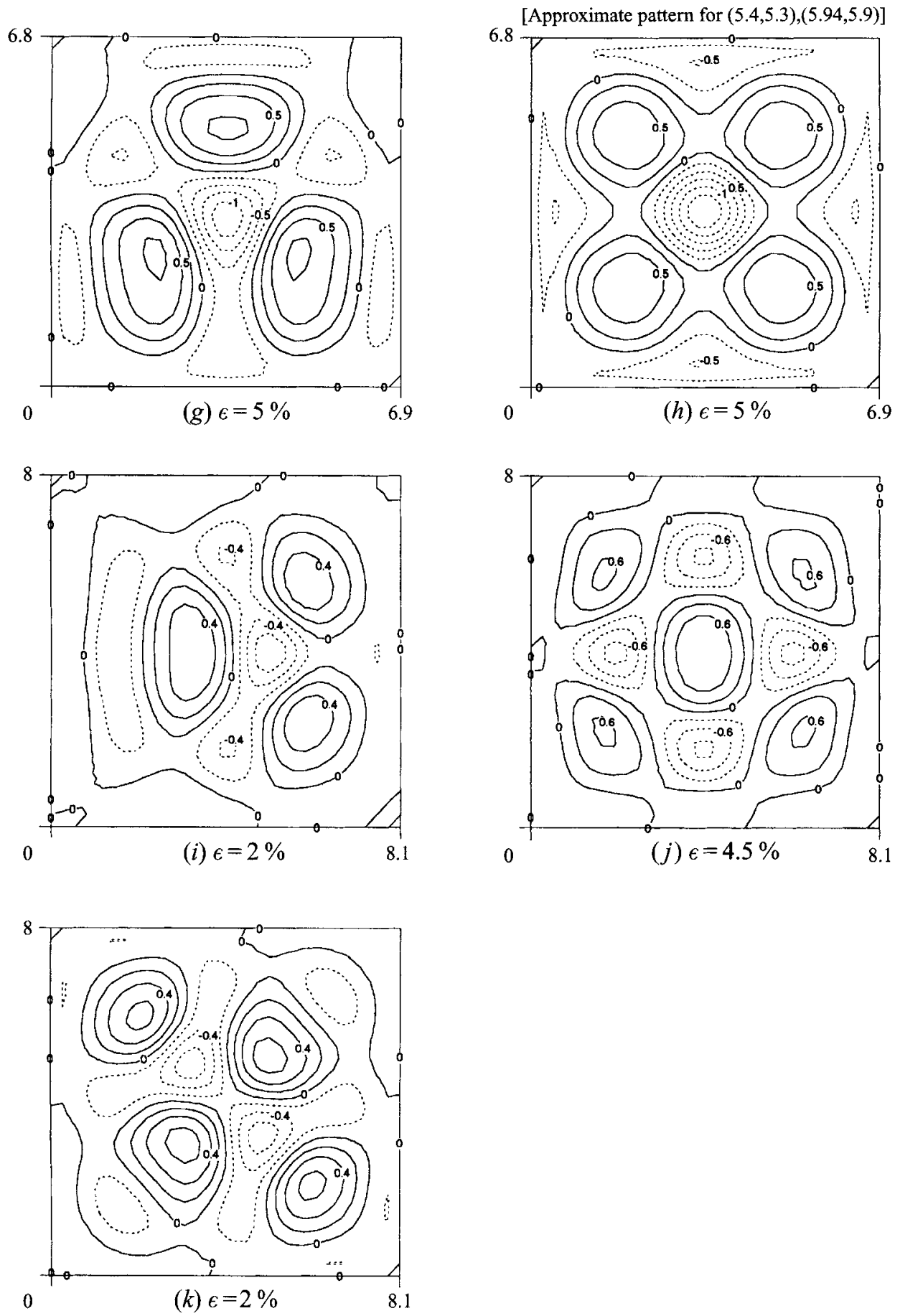

FIGURE 12. Flow patterns in the nonlinear regime for quasi-square containers $(R a=B i=0$, $\left.\operatorname{Pr}=10^{4}\right)$. The iso-values of the vertical velocity $w$ at mid-depth of the fluid layer are represented. The letter under each picture refers to corresponding points in the bifurcation diagrams. The plots $(d),(e)$, $(f)$ and $(h)$ also give approximate representations of the flow for the points labelled $(d),(e),(f)$ and (h) in bifurcation diagrams for boxes with different dimensions. 

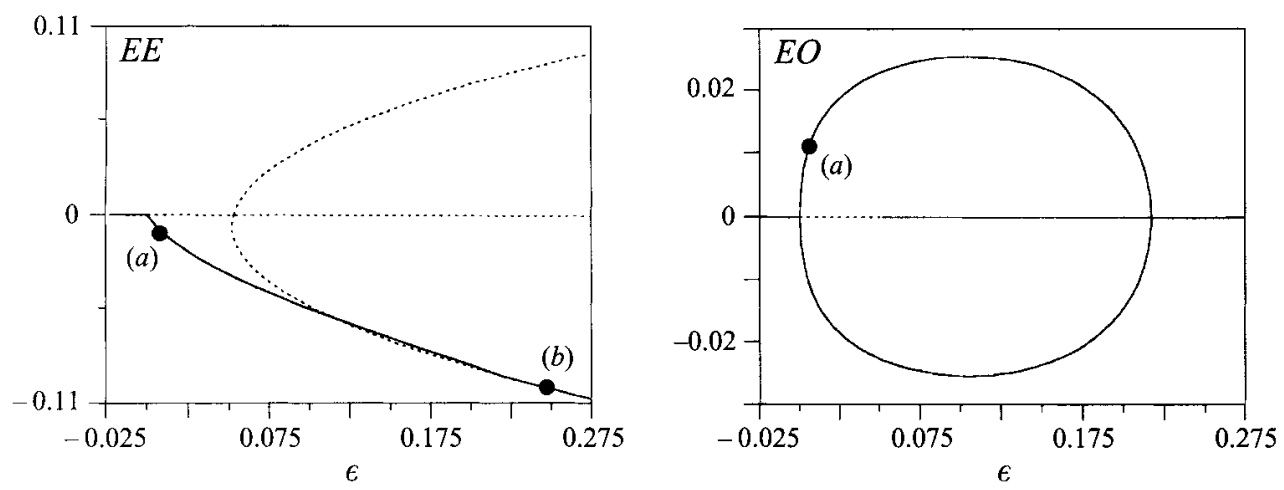

FIGURE 13. Bifurcation diagram for a rectangular box of aspect ratios $\left(a_{1}, a_{2}\right)=(3.2,1.5)$.

the $E O$ and $O E$ modes is of course to be related to the near-degeneracy of the corresponding eigenvalue.

(e) Quasi-square container with aspect ratios $\left(a_{1}, a_{2}\right)=(8.1,8)$

With aspect ratios $\left(a_{1}, a_{2}\right)=(8.1,8)$, the parameters $\left(N_{x}, N_{y}, N_{z}\right)=(7,7,5)$ have turned out to be inappropriate for giving a good approximation of all eigenmodes with growth rate larger than -12 . This is because many eigenmodes with different horizontal structures are nearly critical in such a large container. To obtain better results, we have increased the number of trial functions used for the horizontal dependence of the unknowns and have taken $\left(N_{x}, N_{y}, N_{z}\right)=(8,8,4)$. The parameter $N_{z}$ has been given the value 4 in order for the calculations to remain within reasonable time limits. Of course, precision is lost in the vertical description of the convective patterns. The bifurcation diagram is given in figure 11 and corresponds to $N_{U}=10+9+9+8$ and $N_{S}=18+16+16+14$. These values are determined by considering the modes which bifurcate for $\epsilon$ less than $20 \%$ and which have a growth rate larger than -10 . Clearly, the bifurcation diagram is less precise than for other boxes and only the most important branches (i.e. which exist in large $\epsilon$-windows) are represented. At $\epsilon=0$, a transcritical bifurcation towards a superposition of $O E$ and $E E$ modes can be observed. The pattern for point (i) is given in figure $12(i)$ : three main cells are observed with a slow upwards motion along the left-hand sidewall. Other stable branches are also displayed in figure 11. The convective structures for points $(j)$ and $(k)$ are represented in figures $12(j)$ and $12(k)$. At point $(j)$, the flow is made up by $E E$ modes only and five convective cells are displayed, with a quasi-square cell in the middle of the container. In figure $12(k)$, the presence of four cells and two small areas of slowly rising fluid in two corners of the box can be observed. This pattern is the superposition of the $O O$ and $E E$ modes.

\subsubsection{Rectangular containers}

Four peculiar rectangular boxes are considered in this paragraph. These boxes (see crosses below main diagonal in figure 2 ) are selected in order to provide a general overview on convection in 'actually' rectangular containers $\left(a_{1}\right.$ and $a_{2}$ are 'quite' different). The first case $\left(a_{1}, a_{2}\right)=(3.2,1.5)$ enables us to study the nonlinear evolution of a unique roll parallel to the longer sides of the box. The next two boxes $\left[\left(a_{1}, a_{2}\right)=(5.4,3.2)\right.$ and $\left.\left(a_{1}, a_{2}\right)=(6.6,3.2)\right]$ are selected to study the competition between three or four rolls parallel to the shorter sides and the two rolls parallel to the longer ones which are nearly unstable at the threshold. The last cavity $\left(a_{1}, a_{2}\right)=(6.6$, 

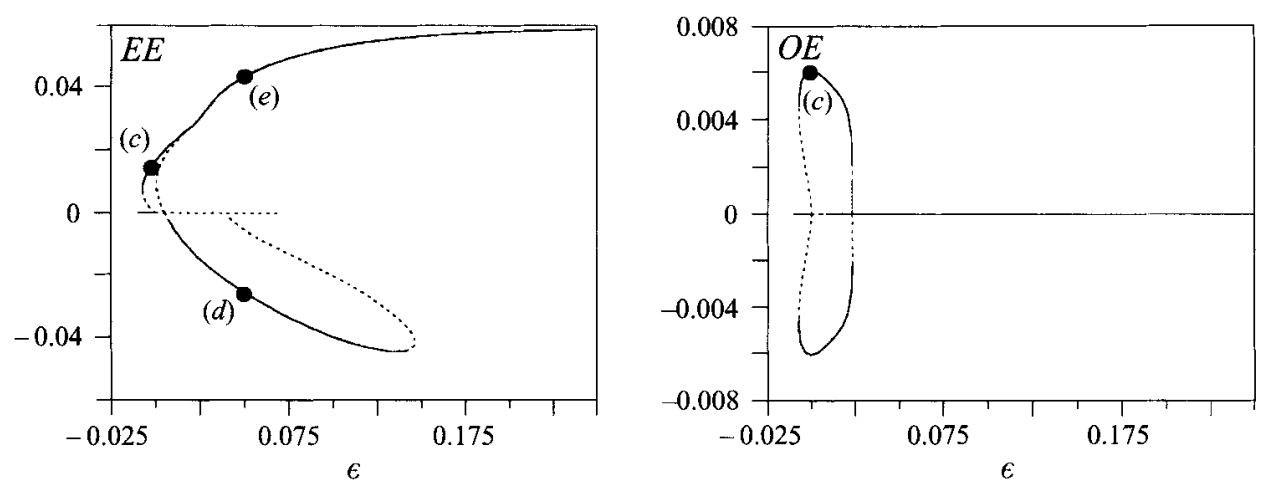

FIGURE 14. Bifurcation diagram for a rectangular box of aspect ratios $\left(a_{1}, a_{2}\right)=(5.4,3.2)$.

5.2) illustrates the evolution of four linearly unstable rolls parallel to the shorter sides when three rolls parallel to the other sides are nearly critical at $M a=M a_{c}$.

(a) Rectangular container with aspect ratios $\left(a_{1}, a_{2}\right)=(3.2,1.5)$

In a rectangular box with aspect ratios $\left(a_{1}, a_{2}\right)=(3.2,1.5)$, the linearly unstable mode belongs to the $E O$ class and the convective pattern at the threshold takes the form of a unique roll parallel to the longer sides of the container. The bifurcation diagram is given in figure 13 and one observes a supercritical bifurcation at threshold. The solution is made up by the superposition of the $E O$ and $E E$ modes generated by the nonlinear evolution of the critical mode. At $\epsilon=22 \%$, the $E E$ solution becomes stable and the $E O$ branch disappears. The patterns corresponding to points $(a)$ and $(b)$ in figure 13 are represented in figures $17(a)$ and $17(b)$, respectively. Figure $17(a)$ clearly shows that the one-roll solution of the linear analysis (figure $5 c$ ) is modified by the presence of the $E E$ modes. In figure $17(b)$, two rolls parallel to the shorter sides of the container are displayed.

(b) Rectangular container with aspect ratios $\left(a_{1}, a_{2}\right)=(5.4,3.2)$

The bifurcation diagram for $\left(a_{1}, a_{2}\right)=(5.4,3.2)$ is plotted in figure 14. A subcritical bifurcation towards the $O E$-solution appears at the threshold. The corresponding pattern (point $c$ ) is represented in figure $17(c)$. This pattern loses stability at $\epsilon=2.3 \%$ where the $E E$ branch becomes stable. The convective pattern on this $E E$ branch (point $e$ ) is formed by two 'horizontally compressed' square cells and is similar to figure $17(e)$ (which corresponds to a box of different aspect ratios). This branch is the only stable solution for $\epsilon$ larger than $14 \%$. The negative part of the $E E$ branch is also stable. The pattern for point $D$ is given in figure $17(d)$ and is made up by two rolls parallel to the longer sides of the box. Note that this solution could not appear in a progressive heating experiment and becomes unstable for $\epsilon$ larger than $14 \%$.

(c) Rectangular container with aspect ratios $\left(a_{1}, a_{2}\right)=(6.6,3.2)$

The linear convective pattern corresponding to $\left(a_{1}, a_{2}\right)=(6.6,3.2)$ consists of four rolls parallel to the shorter sides of the container. The bifurcation diagram for this box is plotted in figure 15. At $\epsilon=0$, there is a transcritical bifurcation. The supercritical branch gives stable solutions for only a very narrow band of $\epsilon$. The stable solution on which the system jumps before the threshold is the negative part of the $E E$ branch. The pattern for this solution (point $e$ ) is represented in figure $17(e)$ where two square cells are observed. These square cells are in fact the superposition of the four linearly 


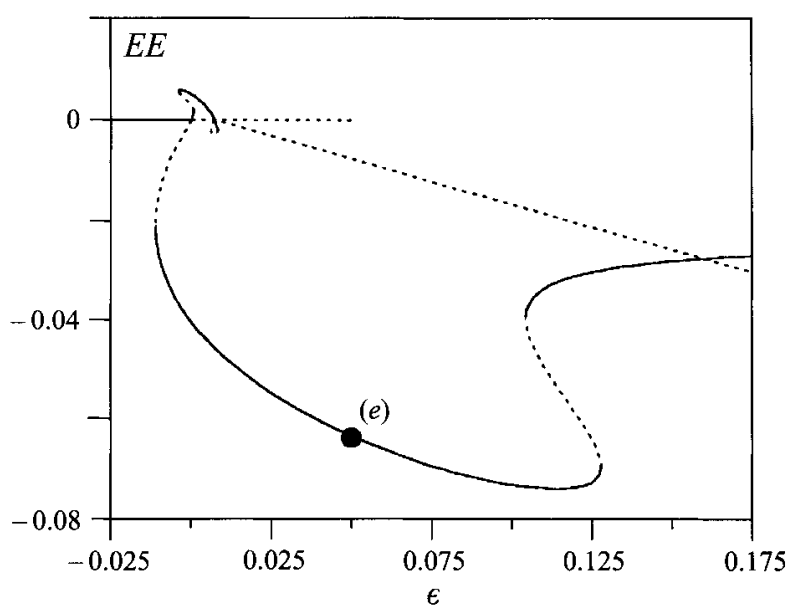

FIGURE 15. Bifurcation diagram for a rectangular box of aspect ratios $\left(a_{1}, a_{2}\right)=(6.6,3.2)$.
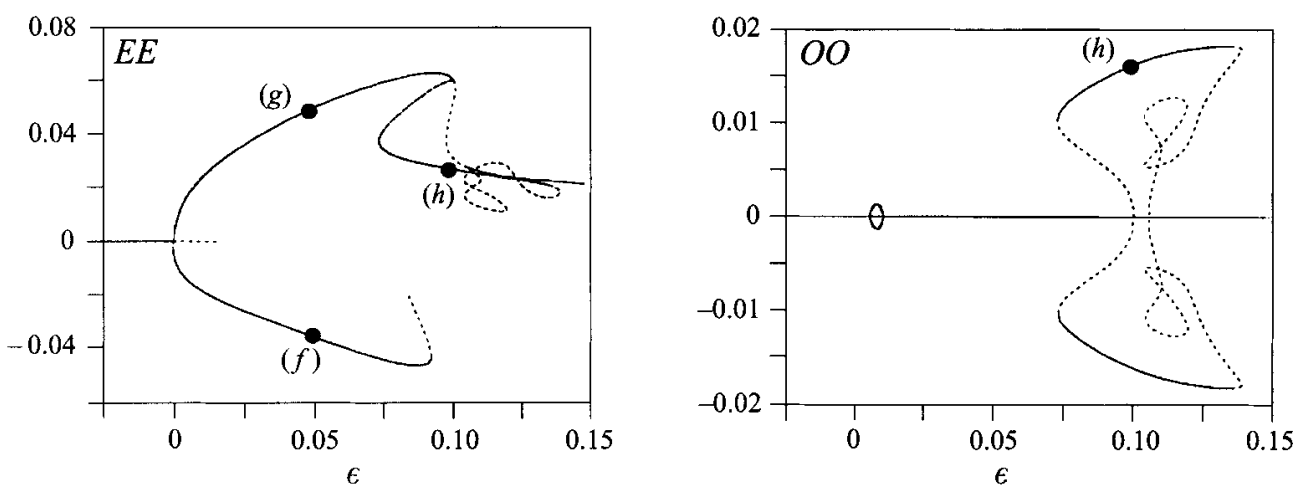

FIGURE 16. Bifurcation diagram for a rectangular box of aspect ratios $\left(a_{1}, a_{2}\right)=(6.6,5.2)$.

unstable rolls parallel to $y$ and the two rolls parallel to $x$ which are nearly critical and generated by the nonlinear evolution of the critical mode.

(d) Rectangular container with aspect ratios $\left(a_{1}, a_{2}\right)=(6.6,5.2)$

Figure 16 is the bifurcation diagram for $\left(a_{1}, a_{2}\right)=(6.6,5.2)$. The bifurcation at the threshold is transcritical but the subcritical domain is nearly vanishing. The pattern for point $(f)$ on the stable negative $E E$ branch (on which the system would jump before reaching the threshold) is that of figure $17(f)$ : four convective rolls parallel to $y$ appear, with upflow in the middle of the container. Rising fluid also appears along the sidewalls parallel to $y$. Figure $17(g)$ represents the motion for point $(g)$ and shows the birth of the four square cells observed in figure $12(h)$. On this branch, a secondary bifurcation gives rise to an $O O-E E$ solution which is represented in figure $17(h)$ for point $(h)$. This picture presents evident similarity with the two-triangular-cells solution of figure $12(d)$.

\subsection{Discussion and comparison with other works}

\subsubsection{Summary of the results}

In $\S 4.3$, we examined Marangoni convection in rigid rectangular boxes within the weakly nonlinear regime. We have studied the stable motions which are likely to be observed for $\epsilon$ extending up to about 15 or $20 \%$. We have not analysed in detail stable 

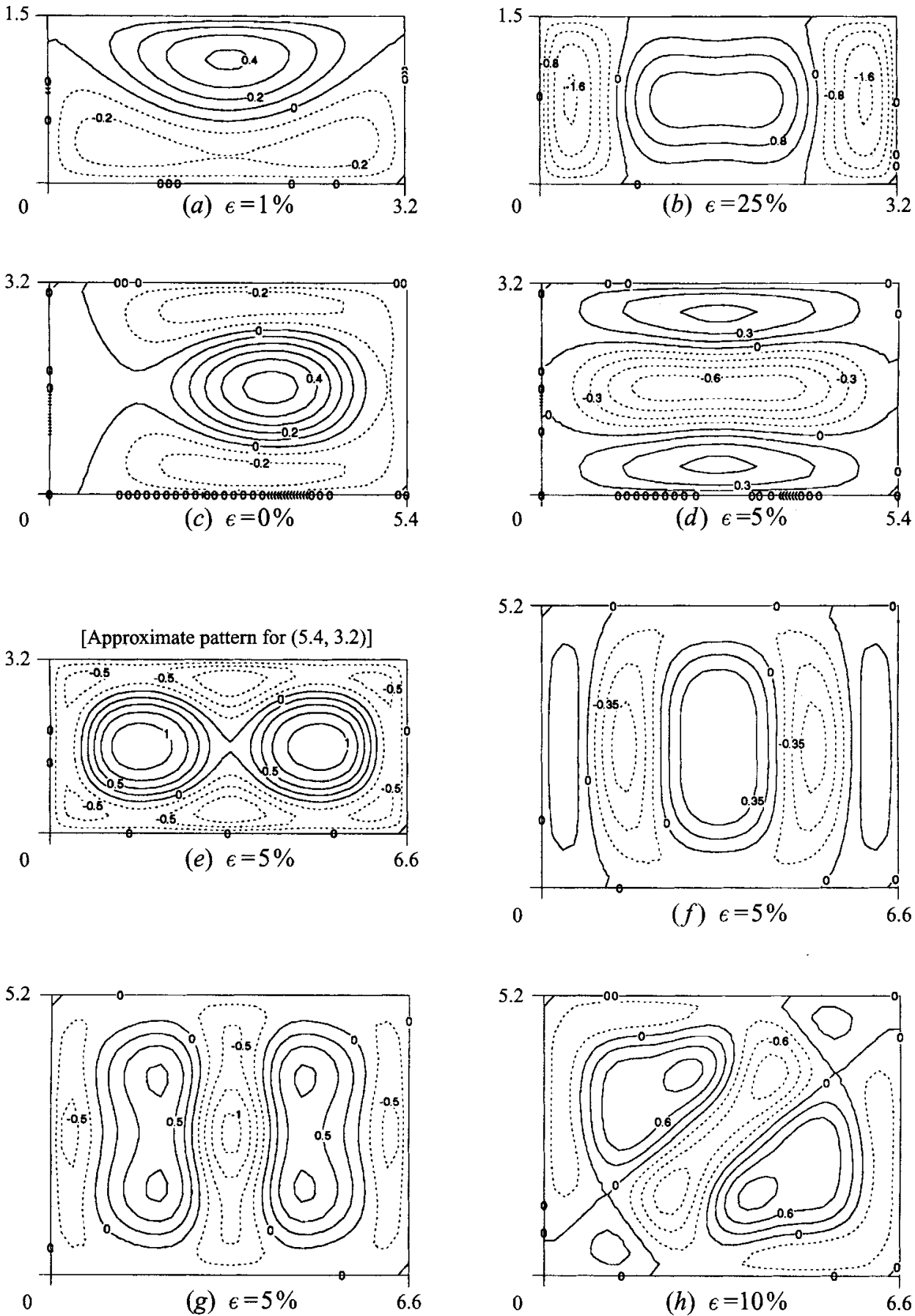

FIGURE 17. Flow patterns in the nonlinear regime for rectangular containers $\left(R a=B i=0, \operatorname{Pr}=10^{4}\right)$. The iso-values of the vertical velocity $w$ at mid-depth of the fluid layer are represented. The letter under each picture refers to the corresponding point in the bifurcation diagrams. The picture $(e)$ also gives an approximate representation of the flow for the point labelled $(e)$ in the bifurcation diagram of figure 14 . 
solutions which exist only over a very narrow window in $\epsilon$ because these solutions could not easily be observed in experiments.

In square containers, the main results are summarized in table 5. For small boxes with aspect ratios between 2.4 and 4.4 , the convective pattern appearing above the threshold is a unique square cell filling up the box and with the fluid flowing up at the centre of the cell. The borders of the cell may be either parallel to the sidewalls (small containers) or form a $45^{\circ}$ angle to the sidewalls (larger boxes). The bifurcation at the threshold is transcritical, which means that the motion sets in with a preferred direction. This property of the primary bifurcation is expected for $E E$ critical eigenmodes and will be observed in all white areas of figure 2.

For the $E O-O E$ region of figure 2 with aspect ratios between 4.6 and 5.6, the stable pattern at threshold is the superposition of $E O$ or $O E$ modes and the $E E$ modes (figure $12 b$ ) but the corresponding structure becomes unstable for rather small values of $\epsilon$. In small containers $\left(a_{1} \approx 4.6\right)$, the unique stable structure is then a square cell. For larger values of $\epsilon$, one has a secondary bifurcation towards a two triangular cells structure. The situation is different in larger containers $\left(a_{1} \approx 5.3\right)$ where several stable structures may coexist for a given value of the distance to threshold. One can observe four square cells ( $E E$ solution), two triangular cells $(O O-E E$ solution) or a three-cell pattern (superposition of eigenmodes of all parities). The three-cell solution consists of a square cell in a corner and two wedge-shaped cells filling symmetrically the remainder of the box. The primary bifurcation point for these $E O$ or $O E$ modes is a pitchfork bifurcation because flows with opposite signs are related by reflection about one of the medians of the box. The bifurcation is subcritical but the subcritical domain is quite small. Recall that in perfectly square boxes, the bifurcation is degenerate.

For a square box with aspect ratio near 5.9 ( $O O$ area in figure 2$)$, the theory predicts the coexistence of the different stable branches corresponding to two, three and four cells. In the immediate neighbourhood of threshold an $E E$ solution with one square cell and rising fluid along the walls is displayed (figure $12 f$ ).

For $6.2 \leqslant a_{1} \leqslant 7.2$, the critical mode has parity $E E$ and the bifurcation is transcritical. For $\epsilon<3.5 \%$, a square cell and a ring of rising fluid along the sidewalls is predicted (figure $12 f$ ). Farther from the threshold, a three-cell solution (figure $12 \mathrm{~g}$ ) and a four-square-cell pattern (figure $12 \mathrm{~h}$ ) are displayed. The form and position of the three cells are, however, completely different from that in smaller boxes. Note also that the triangular cell pattern has disappeared and that the four square cells are the only stable solution for $\epsilon$ larger than about $11 \%$.

In a box of aspect ratio 8 , a stable five-cell solution is found with a central square cell whose borders form a $45^{\circ}$ angle with the sidewalls. A four-cell pattern and a threecell solution with an additional upflow along a sidewall and another $E E-O O$ structure are also observed.

It can thus be stated that the number of cells in square boxes increases progressively with the aspect ratio but it is also important to emphasize that solutions with different numbers of cells usually coexist in large enough vessels (see table 5). Note also the evolution in figures $12(\mathrm{~b}), 12(\mathrm{~g})$ and $12(\mathrm{i})$ which correspond to superpositions of $E E$ and $E O$ or $O E$ modes. In figure $12(b)$, one main cell appears with two small upflows in two corners. In figure $12(\mathrm{~g})$, these upflows have grown to give rise to two cells symmetric with respect to a median. In figure $12(i)$, an additional upflow area along a wall is observed.

Concerning the rectangular boxes, we have only examined some particular aspect ratios. In a container for which the linearly unstable pattern is a roll parallel to the longer sides of the box, we saw that this structure is progressively transformed when 


\begin{tabular}{|c|c|c|c|c|}
\hline $\begin{array}{l}\text { Aspect } \\
\text { ratio }\end{array}$ & Convective pattern & $\begin{array}{l}\text { View } \\
\text { of the } \\
\text { pattern } \\
\text { in figure }\end{array}$ & $\begin{array}{l}\text { Corresponding } \\
\text { experiment } \\
\text { pattern } \\
\text { (Koschmieder \& } \\
\text { Prahl 1990) } \\
\text { in figure }\end{array}$ & $\begin{array}{c}\text { Range of } \epsilon \\
\text { (in } \%)\end{array}$ \\
\hline 2.4 & $1 \mathrm{~S}$ & $12(a)$ & $18(a)$ & $-8.3 \rightarrow$ \\
\hline 4.1 & $1 \mathrm{~S}_{45^{\circ}}$ & $12(c)$ & - & $-1.0 \rightarrow$ \\
\hline \multirow[t]{3}{*}{4.6} & $\mathrm{H}(1$ main $\mathrm{C})$ & $12(b)$ & - & $-0.0 \rightarrow 2.5$ \\
\hline & $1 \mathrm{~S}_{45^{\circ}}$ & $12(c)$ & - & $2.5 \rightarrow 10$ \\
\hline & $2 \mathrm{~T}$ & $12(d)$ & $18(b)$ & $10 \rightarrow$ \\
\hline \multirow[t]{4}{*}{5.3} & $\mathrm{H}$ & $12(b)$ & - & $-0.0 \rightarrow 8.0$ \\
\hline & $2 \mathrm{~T}$ & $12(d)$ & $18(b)$ & $3.0 \rightarrow$ \\
\hline & $4 S$ & $12(h)$ & $18(d)$ & $5.8 \rightarrow$ \\
\hline & $3 C(1 S+2 W)$ & $12(e)$ & $18(c)$ & $6.0 \rightarrow 13$ \\
\hline \multirow[t]{4}{*}{5.9} & $1 \mathrm{~S}+1 \mathrm{R}_{\mathrm{U}}$ & $12(f)$ & - & $-0.0 \rightarrow 3.7$ \\
\hline & $2 \mathrm{~T}$ & $12(d)$ & $18(b)$ & $0.2 \rightarrow 16$ \\
\hline & $3 \mathrm{C}(1 \mathrm{~S}+2 \mathrm{~W})$ & $12(e)$ & $18(c)$ & $3.1 \rightarrow 15$ \\
\hline & $4 \mathrm{~S}$ & $12(h)$ & $18(d)$ & $4.2 \rightarrow 22$ \\
\hline \multirow[t]{3}{*}{6.8} & $1 \mathrm{~S}+1 \mathbf{R}_{\mathrm{U}}$ & $12(f)$ & - & $-0.3 \rightarrow 3.5$ \\
\hline & $13 \mathrm{C}$ & $12(g)$ & - & $0.4 \rightarrow 11$ \\
\hline & $4 \mathrm{~S}$ & $12(h)$ & $18(d)$ & $3.4 \rightarrow 15$ \\
\hline \multirow[t]{3}{*}{8.0} & $\mathrm{H}(3$ main $\mathrm{C})$ & $12(i)$ & - & $0.0 \rightarrow 3.7$ \\
\hline & $\mathrm{H}(4$ main $\mathrm{C})$ & $12(k)$ & $18(e)$ & $1.0 \rightarrow 8.1$ \\
\hline & $5 \mathrm{C}$ & $12(j)$ & - & $4.2 \rightarrow 11$ \\
\hline
\end{tabular}

TABLE 5. Stable convective patterns in quasi-square containers. The first column gives the approximate aspect ratio. The kind of structure is described in the second column ( $S$ : square cell with upflow in the centre, index $45^{\circ}$ : the borders of the cell form a $45^{\circ}$ angle to the walls, $\mathrm{C}$ : cell, $\mathrm{H}$ : hybrid cell, $\mathrm{T}$ : triangular cell, $\mathrm{W}$ : wedge-shaped cell, $\mathrm{R}_{\mathrm{U}}$ : upflow ring along the walls). Possible coexistences of solutions are indicated by vertical lines. The last column gives the range of $\epsilon$ in which the branches corresponding to the different structures are stable.

$\epsilon$ is increased into rolls parallel to the other sides (figure $17 a, b$ ). In the general case where rolls parallel to the shorter sides of the containers are linearly unstable, several cases were analysed. For $\left(a_{1}, a_{2}\right)=(5.4,3.2)$, three rolls are linearly unstable. The primary bifurcation of the $O E$ mode is subcritical but this structure becomes unstable for rather small $\epsilon$ and a two square cells structure appears (figure 17e). A pattern with two rolls parallel to the longer sides is also stable but could not be reached if the temperature gradient is progressively increased from the conductive state. For $\left(a_{1}, a_{2}\right)=(6.6,3.2)$, the motions starts, before threshold, by a jump onto the branch corresponding to two square cells. These square cells are the superposition of the four linearly unstable rolls and the two rolls parallel to the longer sides which bifurcate quite near threshold and are generated by the nonlinear evolution of the critical mode. In a box with $\left(a_{1}, a_{2}\right)=(6.6,5.2)$, the critical eigenmode consists of four rolls parallel to $y$. This structure remains stable in the weakly nonlinear regime with either upflow or downflow at the centre of the box. The structure with upflow at the centre (and along the sidewalls) is, however, more likely to appear, owing to the transcritical character of the primary bifurcation. The pattern with downflow at the centre shows the birth of the four square cells structure. In this box, the two square cells are not observed any more. A secondary bifurcation towards an asymmetric solution (figure 17h) is also displayed. The pattern in this case is similar to the two triangular cell solution obtained in square boxes. 


\subsubsection{Modal interactions and comparison with Rosenblat et al. (1982b)}

It is interesting to study the modal interactions in order to better understand the role of rigid lateral walls on convection. This discussion allows us also to emphasize the difference between the present work and that of Rosenblat et al. (1982b) who used the approximation of slippery sidewalls.

A very important consequence of the presence of rigid walls is that the quadratic selfinteraction of a mode generates all the $E E$ modes, and not only some of them as for slippery walls. If one considers for instance a three-roll marginally stable eigenmode, its nonlinear self-interaction generates the six-roll eigenmode in slippery boxes while, in a rigid box, all the eigenmodes with an even number of rolls $(2,4,6,8, \ldots)$ are excited. This is a consequence of the rigidity of the sidewalls because the vanishing velocity at the walls gives rise to a component of the motion in the whole box and the self-interaction of this component may generate all even solutions. A direct consequence of this property is the transcritical character of the bifurcations of the $E E$ modes. Indeed, the quadratic self-interaction of an $E E$ mode re-generates this $E E$ mode and a quadratic term appears in the evolution equation of the amplitude of this mode. On the other hand, the primary bifurcation points are pitchforks in slippery containers. This difference can easily be understood. Consider for instance two rolls becoming unstable in a slippery box. Since the boundary conditions along the sidewalls parallel to the rolls and the boundary conditions at the border between the rolls are the same, the direction of the flow within the system makes no difference. In contrast, owing to the vanishing of the velocity on the walls, it is not equivalent, in a rigid box, to have a two-roll pattern with upflow at the centre and downflow along the walls or to have motion of the opposite sign. This proves clearly that the absence of transcritical bifurcations for even solutions in an artefact of the slippery walls assumption.

The importance of rigid walls may also be emphasized by reconsidering the container with aspect ratios $\left(a_{1}, a_{2}\right)=(6.6,3.2)$. We know from the linear analysis that the convective structure at threshold is made up of four rolls parallel to the shorter sides of the container. In a rigid box, the vanishing velocity at the ends of the four rolls gives rise to motion along the axes of the rolls and the quadratic self-interaction of this motion may then generate the two rolls parallel to the longer sides so that the setting of square cells is possible. With slippery walls, a four-roll mode generates a mode with eight rolls parallel to the shorter sides of the box but it does not excite the two rolls parallel to the other sides. When the interactions between the two-roll and the four-roll modes are taken into account in slippery boxes, one obtains amplitude equations taking the form of equations (5.15) of Rosenblat et al. (1982b), with no quadratic terms. The corresponding bifurcation diagram is given in figures 10 or 11 of Rosenblat et al. and could not yield square cells directly as in our figure 15, but only after a secondary bifurcation. This example also stresses the importance of the threedimensional effects generated by rigid sidewalls which are absent in slippery boxes.

A direct comparison of our work and that of Rosenblat et al. is not an easy task since these authors present only some results above threshold. Rosenblat et al. examine the problem of nonlinear convection only in very small containers with $a_{2}=1$ and $a_{1}$ smaller than about four. As an example, we have considered the competition between a one-roll solution and a two-roll solution in a rigid box to compare our bifurcation diagram with figures 8 and 9 of Rosenblat et al. We have found rather similar bifurcation diagrams and the only difference was the transcritical bifurcation of our two-roll mode, with a very small subcritical domain. This similarity in the case of very small cavities originates in the fact that the nonlinearly generated modes are quite 
damped in both approaches. The more realistic case of larger vessels was not considered by Rosenblat et al. and a direct comparison is thus not possible. However, it is our opinion that the slippery walls assumption is not very appropriate for describing convection in boxes because the modes which are generated in the nonlinear regime as well as their interactions are quite different from those observed in the case of realistic rigid walls. In particular, weakly damped modes are generated in (large) rigid boxes which are not present in slippery containers.

Another consequence of these modal interactions in rigid boxes is the difference between the convective structures at threshold and in the nonlinear regime. In slippery boxes, the modes which are generated by the nonlinear interactions of the marginally stable modes are strongly damped. As a consequence, their amplitudes remain quite small and do not influence greatly the convective structure. In rigid boxes, one observes the emergence of some modes which are nearly critical and these are thus not well damped. These modes may thus not be adiabatically eliminated. Their amplitude is not small and they can greatly modify the convective structure, even in the immediate neighbourhood of threshold. This result is clearly illustrated by comparing figures 6 and 12 or figures 5 and 17 . Note also that the difference between the linear and nonlinear structures is still reinforced by the fact that the bifurcations are transcritical or subcritical (in sufficiently large containers). For this reason, convection always sets in with finite amplitude in rigid boxes.

\subsubsection{Comparison with the experiments of Koschmieder \& Prahl (1990)}

It is also important to compare our results with experimental observations. In that respect, nice experiments have been performed by Koschmieder \& Prahl (1990) in square boxes. It is, however, worth stressing that, for several reasons, the comparison is mainly qualitative. First, the experiments of Koschmieder \& Prahl have been carried out on Earth so that gravity effects are not strictly absent. Moreover, the experimental Biot numbers are different from zero: they are of the order of 1.9 for small containers and 0.35 for larger ones (Dauby 1994). Finally, the Prandtl number for the silicone oil used in the experiments is about $10^{3}$ while the Prandtl number used in our calculations is $10^{4}$. Nevertheless, we think that a comparison between theory and experiments is relevant because, as shown by the linear analysis, the values of the Rayleigh and Biot numbers are not of great importance for the nature of the convective structure appearing at threshold. Regarding the Prandtl numbers, we think that both values are compatible with an infinite Prandtl number hypothesis. Note also that, since the experimental critical parameters are not known very accurately (errors are of the order of $15 \%$ ), we shall not compare the experimental and theoretical values of these quantities and we shall focus on the convective patterns. In view of the above remarks, we obtained very good agreement with Koschmieder \& Prahl's experiments. Indeed, all the convective patterns observed by these authors in square boxes with aspect ratios smaller than eight (see reproduction of their results in figure 18) were found as stable solutions of our model (table 5). For a square container of aspect ratio 2.4 (figure 12a), the convective structure predicted by our approach is quite similar to the pattern observed by Koschmieder \& Prahl in a square box of aspect ratio 1.82 (figure 18a), with a unique square cell in which the fluid rises at the centre. Our figure $12(d)$ for a square box of aspect ratio 5.9 is also very similar to the two triangular cells obtained by these authors in a box of aspect ratio 5.68 (figure $18 b$ ), with two main upflow areas in the middle of the cells and downflow along the diagonal. The three-cell, four-squarecell and five-cell patterns observed in boxes of aspect ratios 6.16, 6.36 and 8.4 (figures $18 c, d$ and $e$ ) are in agreement with the structures presented in figures $12(e), 12(h)$ and 
(a)

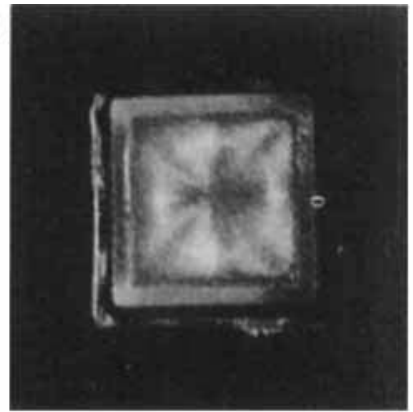

(c)

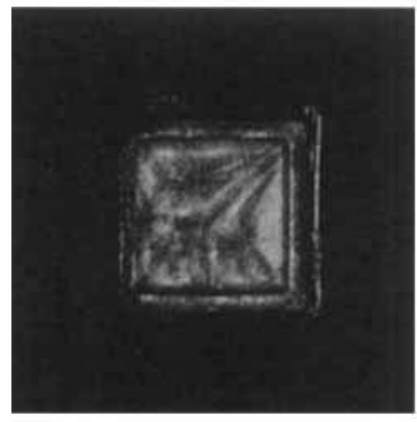

(e)

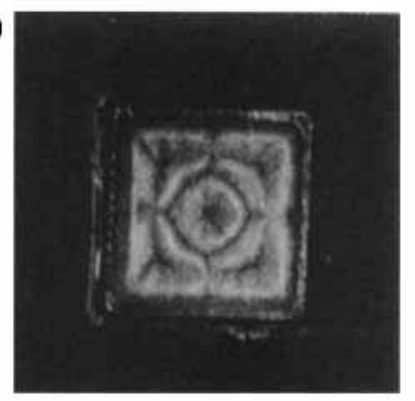

(b)

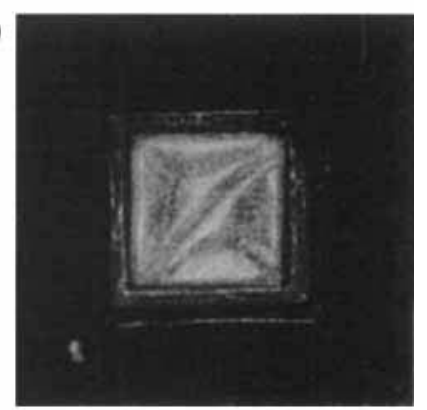

(d)

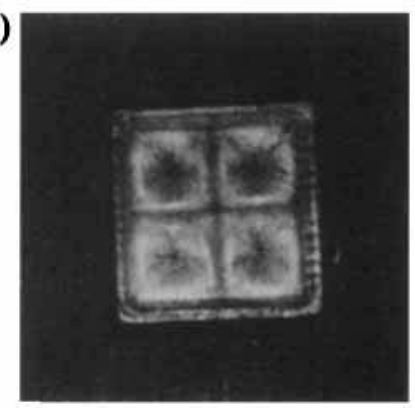

(f)

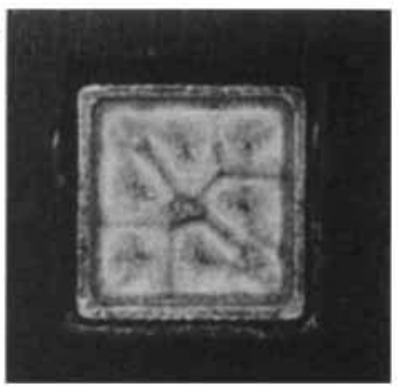

(g)

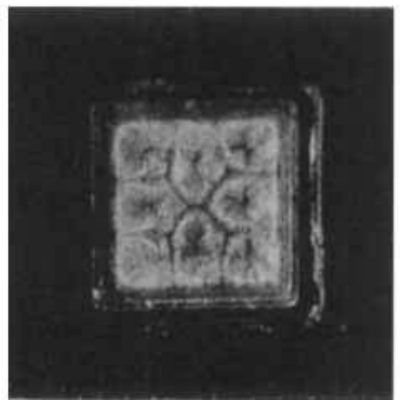

Figure 18. Reproduction of figure 2 of Koschmieder \& Prahl (1990): patterns in a square container. (a) One-cell solution: $M a=380, R a=228, a_{1}=1.82$. (b) Two-cell solution: $M a=54, R a=33$, $a_{1}=5.68$. (c) Three-cell solution: $M a=80, R a=42, a_{1}=6.18$. (d) Four-cell solution: $M a=78$, $R a=38, a_{1}=6.36$. (e) Five-cell solution: $M a=67, R a=19, a_{1}=8.4$. $(f)$ Six-cell solution: $M a=72, R a=22, a_{1}=8.08$. $(g)$ Eight-cell solution: $M a=63, R a=16, a_{1}=8.75$. 
12(i). Note also that, as in our theoretical model, Koschmieder \& Prahl observed multiple stable patterns. For instance, in a box of aspect ratio 8.08 , they saw either five or six convective cells. Finally, it is interesting to mention that the similarity between the observations of Koschmieder \& Prahl and our theoretical predictions usually occurs not very near threshold (table 5). The patterns of Koschmieder \& Prahl are thus probably not critical structures.

On the other hand, it is interesting from an experimental point of view to estimate the magnitude of the velocity in the weakly nonlinear regime. For a relative distance to threshold equal to about $5 \%$, the maximum vertical velocity at mid-depth of the layer is usually of the order of 1 in non-dimensional units (figures 12 and 17). For a $1 \mathrm{~mm}$ deep layer of DC200 Silicone oil (Koschmieder \& Biggerstaff 1986), this corresponds to a velocity of about $10^{-1} \mathrm{~mm} \mathrm{~s}^{-1}$, which is actually in the range of current observations.

\subsubsection{Comment on Dijkstra's $(1995 b, c)$ papers}

The present results may also be compared with the numerical work of Dijkstra (1995b) which contains a nonlinear approach to Marangoni instability based on numerical algorithms. Dijkstra's method is not limited to the weakly nonlinear regime as in our work and should probably deal more easily with systems far from threshold. A direct comparison with Dijkstra's analysis is not easy because we do not consider containers with exactly the same aspect ratios. Nevertheless, we have found that figure 3 for $a_{1}=4$ in Dijkstra $(1995 b)$ is quite similar to the bifurcation diagram that we obtain for $\left(a_{1}, a_{2}\right)=(4.2,4.1)$, with the same stable convective pattern and the same order of magnitude for the velocity. In larger vessels, the agreement is less satisfactory, even though we recover several convective patterns presented by Dijkstra. For a container with aspect ratio 6 (figures 5 and 6 in Dijkstra 1995b), our results are at variance because, as already mentioned in $\S 3.2$, Dijkstra did not consider the proper critical eigenmode. Moreover, we think that the bifurcation of the mode represented in figure $6(b)$ of Dijkstra is not a degenerate one, as claimed by the author. These are probably the reasons why Dijkstra did not obtain the branch originating at point $a$ of his figure 5 .

In a third paper, Dijkstra $(1995 c)$ extends the work described above by studying the formation of hexagonal convective patterns in large square boxes (aspect ratios between 7 and 13). Comparison with this study is not possible at the present stage of our study because our calculation codes are limited to boxes with aspect ratios smaller than about eight.

\section{Conclusions}

Marangoni instability in rectangular containers with realistic rigid walls has been investigated. Both the linear and the weakly nonlinear problems have been discussed for a large Prandtl number $\left(\mathrm{Pr}=10^{4}\right)$. One of the main successes of the present analysis is a theoretical interpretation of Koschmieder \& Prahl's experiments. We have been able to recover all the convective patterns observed by these authors for aspect ratios smaller than eight. The experimental patterns with six or eight cells (figures $18 f$ and $18 \mathrm{~g}$ ) could also probably be obtained from our model but this would require rather expensive calculations. As a continuation of this work, it is intended to reproduce the above analysis in circular containers (see Zaman \& Narayanan 1996 for a linear study) and to consider the influence of non-vanishing Rayleigh and/or Biot numbers in the nonlinear regime. It would also be interesting to examine the influence of the 
Prandtl number. In particular, the change of direction of the flow for very small $\mathrm{Pr}$ predicted in hexagonal convection (Dauby et al. 1993; Parmentier et al. 1966) should also be checked in finite rigid containers. Other new results have also been obtained for rectangular containers and experimental observations would be welcome.

This text presents research results of the Belgian Interuniversity Poles of Attraction (P.A.I. no. 21) initiated by the Belgian State, Prime Minister's Office, Science Policy Programming. The scientific responsibility is assumed by its authors. It is a pleasure to thank our colleagues P. Parmentier and V. Regnier for interesting comments and also Professor H. A. Dijkstra from Utrecht University. We are also grateful to Professor J.-C. Legros and his group (Brussels University) and to the referees whose remarks have contributed to the improvement of this work. Financial support from the European Communities under grant ERB-CHRXTC 940481 is acknowledged. We finally want to thank SEGI (University of Liège) which allowed us to use their IBM9121 and SP2 computers for our calculations.

\section{Appendix A}

In this appendix, we present the numerical method used to solve the linear eigenvalue problem. For computations convenience, the following coordinate transformation is performed:

$$
x^{\prime}=2 x / a_{1}-1, \quad y^{\prime}=2 y / a_{2}-1, \quad z^{\prime}=2 z-1,
$$

which allows us to replace the real fluid volume by the cube $[-1,1] \times[-1,1] \times[-1,1]$. As a consequence, the components of the nabla operator are now $\nabla=\left(2 / a_{1} \partial / \partial x\right.$, $2 / a_{2} \partial / \partial y, 2 \partial / \partial z$ ) while the boundary conditions are expressed at $x, y, z= \pm 1$.

The trial functions which appear in (3.4) are chosen as

$$
\begin{gathered}
u_{i j k}^{X}=a_{1} f_{i}(x) V_{j}(y) \partial_{z} g_{k}(z), \\
v_{i j k}^{X}=0, \\
w_{i j k}^{X}=-\partial_{x} f_{i}(x) V_{j}(y) g_{k}(z), \\
u_{i j k}^{Y}=0, \\
v_{i j k}^{Y}=a_{2} V_{i}(x) f_{j}(y) \partial_{z} g_{k}(z), \\
w_{i j k}^{Y}=-V_{i}(x) \partial_{y} f_{j}(y) g_{k}(z), \\
\theta_{i j k}=m_{i}(x) m_{j}(y) n_{k}(z),
\end{gathered}
$$

where $\partial_{x}, \partial_{y}$ and $\partial_{z}$ denote derivatives with respect to $x, y$ and $z$ respectively. The definite expressions for the functions $f, V, g, m$ and $n$ are given below. It is easily verified that, with the choice (A 2)-(A 8), the incompressibility condition (3.1) is automatically fulfilled.

Because of the symmetry between the 'length' and the 'width' of the box, the same set of trial functions is used to define the $x$ - and $y$-dependences of the velocity component $u$ and the $y$ - and $x$-dependences of the velocity component $v$. Similarly, the $x$ - and $y$-dependences of the temperature are given in terms of a single set of functions $m_{i}$.

Since the box and boundary conditions are symmetric with respect to the planes $x=0$ and $y=0$, it is sufficient to consider horizontal trial functions $f, V$ and $m$ which 
are either symmetric or antisymmetric with respect to these planes, that is even or odd horizontal trial functions with respect to $x$ or $y$. Because of equations (3.2) and (3.3), the functions $w, \theta, \partial u / \partial x$ and $\partial v / \partial y$ have the same parity in $x$ and $y$ and the classification of the trial functions may be done in terms of the parity of the $m_{i}$ only. The four different classes of solutions will be referred to as the $O O, O E, E O$ and $E E$ solutions. The first and second letter of each symbol refers to the parity of the $x$ - and $y$-dependences of the horizontal temperature trial functions respectively. These letters $E$ and $O$ also characterize the parity of the number of rolls making up the solution and normal to the $x$-and $y$-axes respectively. Consider for instance an even function $m_{i}(x)$. The corresponding $f_{i}(x)$ are odd functions and the sign of the horizontal velocity component $u$ is changed an odd number of times along the $x$-axis. This gives rise to an even number of rolls normal to $x$. It follows that knowledge of the class of the critical solution only provides some preliminary information about the pattern of the convective cells at threshold.

The functions $f, V, G, m$ and $n$ are selected in order that (A 2)-(A 8) form a complete set but also in such a way that most of the boundary conditions are a priori satisfied. These requirements may be satisfied by considering products of Chebyshev polynomials and other polynomials allowing to take the boundary conditions into account. Explicitly, one has

$$
\begin{aligned}
& f_{i}(\zeta)=\left(\zeta^{2}-1\right)^{2} T_{i-1}(\zeta), \\
& V_{i}(\zeta)=\left(\zeta^{2}-1\right) T_{i-1}(\zeta), \\
& g_{k}(z)=(z+1)^{2}(z-1) T_{k-1}(z), \\
& m_{i}(\zeta)= \begin{cases}\int_{0}^{\zeta}\left(\xi^{2}-1\right) T_{2 j}(\xi) \mathrm{d} \xi & \text { for } \quad i=2 j+1 \\
1 & \text { for } i=2 \\
\int_{0}^{\zeta}\left(\xi^{2}-1\right) T_{2 j-3}(\xi) \mathrm{d} \xi & \text { for } \quad i=2 j(j>1),\end{cases} \\
& n_{k}(z)=(z+1) T_{k-1}(z),
\end{aligned}
$$

where $\zeta$ stands for $x$ or $y$. The $T$ are the Chebyshev polynomials. Even (odd) indices $i$ correspond to odd (even) $f_{i}$ or $V_{i}$ functions and even (odd) $m_{i}$.

With this choice for the trial functions, it is easy to verify that the zero-velocity condition on rigid walls, the adiabaticity conditions on the lateral walls as well as the fixed temperature condition at the bottom of the box are automatically satisfied. The vanishing of $w$ at the top surface is also ensured and only the Marangoni and Biot conditions are not $a$ priori verified by the trial functions.

The spectral Tau method consists in introducing the general form (3.4) with (A 2)-(A 13) in (3.2)-(3.3) and boundary conditions (2.10)-(2.11). The algebraic equations are obtained by projection on the trial functions, that is by multiplying the equations by the trial functions and integrating the products over the fluid volume.

First, the field equations (3.2)-(3.3) are projected on $\left(v_{p q r}^{X}, 0\right)$ for $p=1, \ldots, N_{x}$, $\mathrm{q}=1, \ldots, N_{y}$ and $r=1, \ldots, N_{z}-1$. This results in

$$
\begin{array}{r}
\left\langle\boldsymbol{v}_{p q r}^{X} \cdot\left[\nabla^{2}\left(\sum_{i, j, k} A_{i j k} \boldsymbol{v}_{i j k}^{X}+\sum_{i, j, k} B_{i j k} \boldsymbol{v}_{i j k}^{Y}\right)+R a\left(\sum_{i, j, k} C_{i j k} \theta_{i j k}\right) \boldsymbol{e}_{z}\right]\right\rangle=0 \\
\text { for } p=1, \ldots, N_{x}, \mathrm{q}=1, \ldots, N_{y}, r=1, \ldots, N_{z}-1,
\end{array}
$$


where $\langle\ldots\rangle$ denotes the integral over the fluid volume. Note that the pressure term has been omitted because, after integrations by parts and use of the a priori satisfied boundary conditions for the velocity, one can check that $\left\langle\boldsymbol{v}_{p q r} \cdot \nabla p\right\rangle=0$. A similar equation is obtained by projecting the field equations on $\left(v_{p q}^{Y}, 0\right)$ for $p=1, \ldots, N_{x}$, $q=1, \ldots, N_{y}$ and $r=1, \ldots, N_{z}-1$.

The Marangoni condition is taken into account by projection of (2.11) on the horizontal velocity trial function at $z=1$ for $p=1, \ldots, N_{x}, q=1, \ldots, N_{y}, r=N_{z}$. One obtains

$$
\begin{array}{r}
\left\langle\left(\begin{array}{c}
u_{p q r}^{X} \\
0
\end{array}\right) \cdot\left[2 \frac{\partial}{\partial z}\left(\sum_{i, j, k} A_{i j k}\left(\begin{array}{c}
u_{i j k}^{X} \\
0
\end{array}\right)+\sum_{i, j, k} B_{i j k}\left(\begin{array}{c}
0 \\
v_{i j k}^{Y}
\end{array}\right)\right)+M a \nabla_{h} \sum_{i, j, k} C_{i j k} \theta_{i j k}\right]\right\rangle_{z=1}=0 \\
\text { for } p=1, \ldots, N_{x}, q=1, \ldots, N_{y}, r=N_{z}
\end{array}
$$

and a similar expression for the projection on the second component of velocity. The following notation is used:

$$
\nabla_{h}=\left(2 / a_{1} \partial / \partial x, 2 / a_{2} \partial / \partial y\right) \text { and }\langle a(x, y, z)\rangle_{z=1}=\int_{-1}^{1} \mathrm{~d} x \int_{-1}^{1} \mathrm{~d} y a(x, y, z=1) .
$$

The field equations (3.2)-(3.3) are also projected on $\left(0, \theta_{p q r}\right)$ for $p=1, \ldots, N_{x}$, $q=1, \ldots, N_{y}$ and $r=1, \ldots, N_{z}-1$. It is found that

$$
\begin{aligned}
\left\langle\theta_{p q r}\left[\nabla^{2}\left(\sum_{i, j, k} C_{i j k} \theta_{i j k}\right)+\sum_{i, j, k} A_{i j k} w_{i j k}^{X}+\sum_{i, j, k} B_{i j k} w_{i j k}^{Y}\right]\right\rangle=0 \\
\quad \text { for } p=1, \ldots, N_{x}, q=1, \ldots, N_{y}, r=1, \ldots, N_{z}-1 .
\end{aligned}
$$

The projection of the Biot condition (2.10) on $\theta_{p q r}$ at $z=1$ and for $p=1, \ldots, N_{x}$, $q=1, \ldots, N_{y}, r=N_{z}$ yields

$$
\begin{aligned}
\left\langle\theta_{p q r}\left[2 \frac{\partial}{\partial z}\left(\sum_{i, j, k} B_{i j k} \theta_{i j k}\right)+B i \sum_{i, j, k} B_{i j k} \theta_{i j k}\right]\right\rangle_{z=1} & =0 \\
\text { for } p & =1, \ldots, N_{x}, q=1, \ldots, N_{y}, r=N_{z} .
\end{aligned}
$$

The set of equations (A 14)-(A 17) forms an algebraic eigenvalue problem for the Marangoni number $M a$ if the Rayleigh number $R a$ is fixed or for the Rayleigh number if $M a$ is fixed. Formally, this system may be written as

$$
\left.\left[\left(\begin{array}{cc}
(A B A B)(A B C) \\
(C A B) & (C C)
\end{array}\right)+\lambda\left(\begin{array}{cc}
\cdot & (L) \\
\cdot & \cdot
\end{array}\right)\right]\left[\begin{array}{c}
A \\
B
\end{array}\right)\right]=0,
$$

where $\lambda$ stands for the eigenvalue parameter ( $s, R a$ or $M a$ ). The first 'row' of equation (A 18) corresponds to (A 14)-(A 15) and the second to (A 16)-(A 17). The quantities $A, B$ and $C$ are vectors whose components are $A_{i j k}, B_{i j k}$ and $C_{i j k}$ respectively. The dimensions of $\boldsymbol{A}, \boldsymbol{B}$ and $C$ are $N=N_{x} \times N_{y} \times N_{z}$ so that the dimension of the eigenvalue problem is $3 \times N_{x} \times N_{y} \times N_{z}$. The numerical calculations have been performed by using the fortran NAG F02BJF procedure.

Let us finally recall that the actual resolution of the eigenvalue problem is carried out by solving four algebraic eigenvalue problems (A 18), which correspond to the four classes of solutions defined above. The critical $M a_{c}$ or $R a_{c}$ characterizing the onset of convection is obtained as the minimum of the four $\lambda$ corresponding to these classes. 


\section{Appendix B}

In this Appendix, we present the equations and boundary conditions of the adjoint linear eigenvalue. These relations can be written as

$$
\begin{gathered}
\nabla^{2} v^{*}-\nabla p^{*}+\theta^{*} e_{z}=\sigma P r^{-1} v^{*}, \\
\nabla \cdot v^{*}=0, \\
\nabla^{2} \theta^{*}+R a w^{*}=\sigma \theta^{*}, \\
v^{*}=\theta^{*}=0 \quad \text { at } z=0, \\
\frac{\partial u^{*}}{\partial z}=\frac{\partial v^{*}}{\partial z}=w^{*}=0 \quad \text { at } z=1, \\
\frac{\partial \theta^{*}}{\partial z}+B i \theta^{*}+M a \frac{\partial w^{*}}{\partial z}=0 \quad \text { at } z=1, \\
v^{*}=\frac{\partial \theta^{*}}{\partial x}=0 \quad \text { at } \quad x=0, a_{1}, \\
v^{*}=\frac{\partial \theta^{*}}{\partial y}=0 \quad \text { at } y=0, a_{2},
\end{gathered}
$$

where the starred quantities refer to the adjoint problem unknown fields.

Let us recall that the direct system (3.1)-(3.3) and its adjoint have the same eigenvalues. Moreover, if both systems are considered as eigenvalue problem for the growth rate $s$, when the Rayleigh and Marangoni numbers are fixed, the following biorthogonality relations between the eigenmodes of both problems are satisfied:

$$
\left(\sigma_{p}-\sigma_{q}\right)\left\langle\theta_{p}^{*} \theta_{q}+\operatorname{Pr}^{-1} \boldsymbol{v}_{p}^{*} \cdot \boldsymbol{v}_{q}\right\rangle=0 .
$$

In this formula, indices $p$ and $q$ characterize eigenmodes of the direct and adjoint problems respectively.

The adjoint system is solved by means of a spectral Tau method, in complete analogy with the direct problem. The normalization condition is also identical.

\section{REFERENCES}

BÉnARd, H. 1900 Rev. Gen. Sci. Pure Appl. 11, 1261.

Canuto, C., Hussaini, M. Y., Quarteroni, A. \& ZANG, T. A. 1988 Spectral Methods in Fluid Dynamics. Springer.

ChandRaseKhar, S. 1961 Hydrodynamic and Hydromagnetic Stability. Clarendon.

DaubY, P. C. 1994 Instabilités thermocapillaires et effets de confinement. PhD thesis, University of Liège, Belgium.

Dauby, P. C. \& Lebon, G. 1994 Series on Advances in Mathematics for Applied Sciences (ed. S. Rionero \& T. Ruggeri), vol. 23, pp. 118-123. World Scientific. (Proc. of the VII Intl Conf. on Waves and Stability in Continuous Media, Bologna, Italy, 4-9 October 1993).

Dauby, P. C., Lebon, G. \& Colinet, P. 1996 Series on Advances in Mathematics for Applied Sciences. World Scientific. (Proc. of the VIII Int. Conf. on Waves and Stability in Continuous Media, Palermo, Italy, 9-15 October 1995).

Dauby, P. C., Lebon, G., Colinet, P. \& Legros, J. C. 1993 Q. J. Mech. Appl. Maths, 46, 683.

DAVIES-Jones, R. P. 1970 J. Fluid Mech. 44, 695.

DAvis, S. H. 1967 J. Fluid Mech.30, 465.

Dijkstra, H. A. 1992 J. Fluid Mech. 243, 73.

Diukstra, H. A. 1995 a Microgravity Sci. Technol. 7, 307. 
Dijkstra, H. A. $1995 b$ Microgravity Sci. Technol. 8, 70.

Dijkstra, H. A. 1995 c Microgravity Sci. Technol. 8, 155.

Eckhaus, W. 1965 Studies in Nonlinear Stability Theory. Springer.

Finlayson, B. A. 1972 The Method of Weighted Residuals and Variational Principles. Academic.

Foias, C., Jolly, M. S., Kevrekidis, I. G., Sell, G. R. \& Titi, E. S. 1988 Phys. Lett. A 131, 433.

HAKEN, H. 1983 Advanced Synergetics. Springer.

Koschmieder, E. L. 1993 Bénard Cells and Taylor Vortices. Cambridge University Press.

Koschmieder, E. L. \& Biggerstaff, M. I. 1986 J. Fluid Mech. 167, 49.

Koschmieder, E. L. \& Prahl, S. A. 1990 J. Fluid Mech. 215, 571.

Lebon, G. \& Pérez-García, C. 1980 Bull. Classe Sci., Acad. R. Belg. 64, 520.

Luijkx, J. M. \& Platten, J. K. 1981 J. Non-equilibr. Therm. 6, 141.

Manneville, P. 1990 Dissipative Structures and Weak Turbulence. Academic.

Nield, D. A. 1964 J. Fluid Mech. 19, 341.

Parmentier, P., Regnier, V., Lebon, G. \& Legros, J.-C. 1996 Phys. Rev. E 53 (to be published).

Pearson, J. R A. 1958 J. Fluid Mech. 4, 489.

Pellew, A. \& Southwell, R. V. 1940 Prov. R. Soc. A 176, 312.

Platten, J. K. \& Legros, J. C. 1984 Convection in Liquids. Springer.

RAYleigh, LoRd 1916 Phil. Mag. 32, 529.

Rosenblat, S., Davis, S. H. \& Homsy, G. M. 1982 a J. Fluid Mech. 120, 91.

Rosenblat, S., Homsy, G. M. \& Davis, S. H. $1982 b$ J. Fluid Mech. 120, 123.

SEYDEL, R. 1988 From Equilibrium to Chaos. Elsevier.

TAKashima, M. 1970 J. Phys. Soc. Japan 28, 810.

VIDAL, A. \& ACrivos, A. 1966 Phys. Fluids 9, 615.

Vooren, A. I. van de \& Dijkstra, H. A. 1989 Comput. Fluids 17, 467.

Winters, K. H. \& Plesser, Th. 1988 Physica D 29, 387.

ZAman, A. A. \& Narayanan, R. 1996 J. Colloid Interface Sci. 179, 151. 